\section{Accepted Manuscript}

Stochastic synchronization of semi-Markovian jump chaotic Lur'e systems with packet dropouts subject to multiple sampling periods

Qian Li, Xinzhi Liu, Qingxin Zhu, Shouming Zhong, Jun Cheng

PII:

DOI: S0016-0032(19)30426-0

Reference: https://doi.org/10.1016/j.jfranklin.2019.06.005 FI 3990

To appear in: Journal of the Franklin Institute

Received date: Revised date: Accepted date:
4 September 2018

26 May 2019

5 June 2019

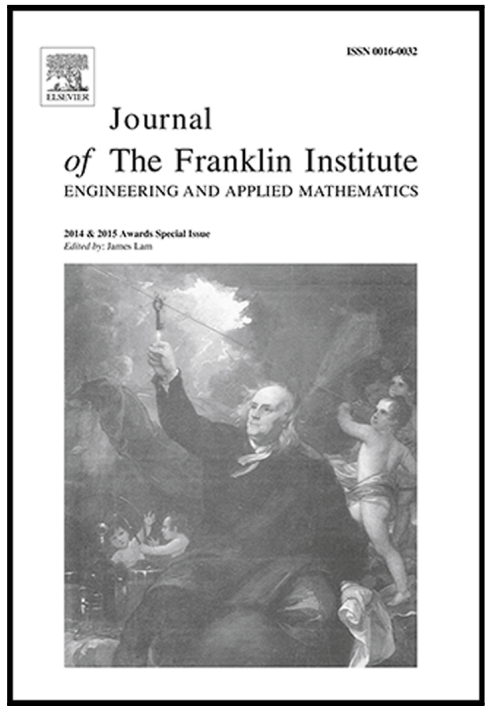

Please cite this article as: Qian Li, Xinzhi Liu, Qingxin Zhu, Shouming Zhong, Jun Cheng, Stochastic synchronization of semi-Markovian jump chaotic Lur'e systems with packet dropouts subject to multiple sampling periods, Journal of the Franklin Institute (2019), doi: https://doi.org/10.1016/j.jfranklin.2019.06.005

This is a PDF file of an unedited manuscript that has been accepted for publication. As a service to our customers we are providing this early version of the manuscript. The manuscript will undergo copyediting, typesetting, and review of the resulting proof before it is published in its final form. Please note that during the production process errors may be discovered which could affect the content, and all legal disclaimers that apply to the journal pertain.

The final publication is available at Elsevier via https://doi.org/10.1016/j.jfranklin.2019.06.005. (C) 2019. This manuscript version is made available under the CC-BY-NC-ND 4.0 license http://creativecommons.org/licenses/by-nc-nd/4.0/ 


\title{
Stochastic synchronization of semi-Markovian jump chaotic Lur'e systems with packet dropouts subject to multiple sampling periods
}

\author{
Qian $\operatorname{Li}^{\mathrm{a}, *}$ Xinzhi Liu ${ }^{\mathrm{b}}$, Qingxin Zhu ${ }^{\mathrm{a}}$, Shouming Zhong ${ }^{\mathrm{c}}$, Jun Cheng ${ }^{\mathrm{d}, \mathrm{e}, *}$ \\ ${ }^{a}$ College of Science, Northwest A\&F University, Yangling 712100, P.R. China \\ ${ }^{b}$ Department of Applied Mathematics, University of Waterloo, Waterloo, Canada \\ ${ }^{c}$ School of Mathematics Sciences, University of Electronic Science and Technology of China, Chengdu 611731, P.R. China \\ ${ }^{d}$ College of Mathematics and Statistics, Guangxi Normal University, Guilin 541006, P.R. China \\ ${ }^{e}$ School of Automation and Electronic Engineering, Qingdao University of Science and Technology, Qingdao 266061, P.R. \\ China
}

\begin{abstract}
This paper is concerned with the problem of stochastic synchronization for semi-Markovian jump chaotic Lur'e systems. Firstly, packet dropouts and multiple sampling periods are both considered. By input-delay approach and then fully considering the probability distribution characteristic of packet dropouts in the modeling, the original system is transformed to a stochastic time-delay system. Secondly, by getting the utmost out of the usable information on the actual sampling pattern, the probability distribution values of stochastic delay taking values in $m$ given intervals can be explicitly obtained. Then, a newly augmented Lyapunov-Krasovskii functional is constructed. Based on that, some sufficient conditions in terms of linear matrix inequalities (LMIs) are derived to ensure the stochastic stability of the error system, and thus, the master system stochastically synchronize with the slave system. Finally, the effectiveness and potential of the obtained results is verified by a simulation example.
\end{abstract}

Keywords: Stochastic synchronization, semi-Markovian jump, chaotic Lur'e systems, sampled-data, packet dropouts

\section{Introduction}

Synchronization, as an important behavior of complex dynamical networks, has received much of the focus [1-3]. Since the pioneering work of [4], the problem of master-slave synchronization for chaotic systems has arisen a great attention. This stems from its potential applications in various fields, including secure communication, image processing, biological systems, chemical reaction, and information science. It has been noted that many nonlinear systems can be represented in the form of Lur'e systems, such as neural networks and Chua's circuits. Thus, the problem of master-slave synchronization of chaotic Lur'e systems

\footnotetext{
* Corresponding author

Email addresses: liqian0210@126.com (Qian Li), jcheng6819@126.com (Jun Cheng)
} 
has been widely studied, and many important results have been proposed(see, [5-8] and the references therein).

Jump systems are a special class of stochastic dynamic systems, have attracted substantial attention from theoretical research to industrial applications [9-23]. Especially, the parameter-switching phenomenon is characterized by a stochastic process in the jump systems. The stochastic process generally relies on the duration $h$ between two consecutive transitions, which is also termed as sojourn-time. In the Markovian jump systems, the sojourn-time $h$ is obeying exponential distribution. In practice, however, it is difficult to guarantee the rigorous restriction on the memoryless characteristics of the sojourn-time distribution. In this case, the underlying continuous stochastic process with sojourn-time obeying non-exponential distribution is often addressed as a semi-Markov process [38,40-44]. It is known that most analysis and design conditions of Markovian jump systems are analytically tractable. Nevertheless, the sojourn-time-dependent characteristics bring much difficulty for the analysis and synthesis of semi-Markovian jump systems, which also results in little presence of numerically solvable synthesis criteria for semi-Markovian jump systems. Thus, it is of great significance to further study the analysis and synthesis of semi-Markovian jump systems, which partially motivates this research.

Nowadays, most of the practical control systems employ the digital signals to transmit the information due to their great advantages over traditional control systems, such as low cost, reduced weight and power requirements, simple installation and maintenance, and high reliability. In the implementation of sampleddata control systems, only the sampled information at its sampling instants is transmitted to the controller, which can reduce the amount of transmitted information and effectively save the communication bandwidth. As a result, sampled-data control strategy has been employed extensively [24-28,39]. The investigation of sampled-data control of chaotic systems is more efficient and useful in real-life applications. Many distinct approaches exist for sample data systems such as lifting technique [29], input delay approach [30], impulsive approach [31], and output delay approach [32], which are used to convert the sampled data output into a continuous time-varying delayed output. The most popular approach is input delay approach, which is based on modeling the sample-and-hold with a delayed control input.

The packet dropout is one of the important issues, which results from transmission errors or congestion in the physical communication links or from buffer overflows [33,34]. It is concluded that packet dropouts degrade system performance and possibly cause system instability. By an input-delay approach, papers [35-37] studies the models with a class of input-delays subject to randomness, where the randomness only comes from the aperiodic sampling period. It should be noticed that, when packet dropouts occur and assuming systems with packet dropouts subject to multiple sampling periods, the input-delay approach will bring input delay double randomness, which comes from not only the randomness of the actual successive packet dropouts, but also the randomness of multiple sampling periods. To the best of our knowledge, there is little information in the published literature about stochastic synchronization of semi-Markovian jump 
chaotic Lur'e systems with packet dropouts subject to multiple sampling periods, which inspires us for this study.

Motivated by the discussions made above, this paper addresses the stochastic synchronization problem of semi-Markovian jump chaotic Lur'e systems. Firstly, the packet dropouts are assumed to subject to multiple sampling periods. Secondly, by getting the utmost out of the usable information on the actual sampling pattern and by fully considering the probability distribution characteristic of packet dropouts and an inputdelay approach, the probability distribution values of stochastic delay taking values in $m$ given intervals can be explicitly obtained. Then, a newly augmented Lyapunov-Krasovskii functional is constructed. Some sufficient conditions in terms of LMIs are derived to ensure the master system stochastically synchronize with the slave system. Finally, an illustrative example is carried out to validate the effectiveness of the developed approach.

Notations: Throughout this paper, the notations used are fairly standard. $\mathbf{N}$ stands for the set of nonnegative integers. $\mathbf{R}^{n}$ denotes $n$-dimensional Euclidean space. For symmetric matrices $X$ and $Y$, the notation $X>Y(X \geq Y)$ means that the matrix $X-Y$ is positive definite (nonnegative). $(\Omega, \mathcal{F}, \mathcal{P})$ is a probability space, where $\Omega$ is the sample space, $\mathcal{F}$ is the $\sigma$-algebra of subsets of the sample space, and $\mathcal{P}$ is the probability measure on $\mathcal{F}$. $E\{\cdot\}$ denotes the expectation operator with respect to some probability measure $\mathcal{P}$. $\|\cdot\|$ denotes the Euclidean norm of a vector and its induced norm of a matrix. The superscripts $T$ and $(-1)$ stand for matrix transposition and matrix inverse respectively. $A \otimes B$ denotes the Kronecker product of the matrices $A$ and $B . A_{s}$ is defined as $A_{s}=\frac{1}{2}\left(A+A^{T}\right)$.

\section{Preliminaries}

Consider the following semi-Markovian jump master system:

$$
\left\{\begin{array}{l}
\dot{x}(t)=A\left(r_{t}\right) x(t)+B\left(r_{t}\right) x(t-h)+W\left(r_{t}\right) f(D x(t)), \\
\gamma(t)=C\left(r_{t}\right) x(t),
\end{array}\right.
$$

where $x(t) \in \mathbf{R}^{n}$ is the state vector of master system, $\gamma(t) \in \mathbf{R}^{l}$ is the output of master system, $h>0$ is constant time delay. It is assumed that $f(\cdot): \mathbf{R}^{m_{f}} \rightarrow \mathbf{R}^{m_{f}}$ is a nonlinear function vector. $A\left(r_{t}\right), B\left(r_{t}\right)$, $C\left(r_{t}\right), W\left(r_{t}\right)$ and $D$ are known real matrices with appropriate dimensions.

Assumption 2.1. $f_{s}(\cdot)$ in (1) is continuous and bounded, and there exist constants $k_{s}^{-}$and $k_{s}^{+}$such that

$$
k_{s}^{-} \leq \frac{f_{s}\left(x_{1}\right)-f_{s}\left(x_{2}\right)}{x_{1}-x_{2}} \leq k_{s}^{+}, \quad s=1,2, \ldots, m_{f}
$$

where $x_{1}, x_{2} \in \boldsymbol{R}$, and $x_{1} \neq x_{2}$.

Let $\left\{r_{t}, t \geq 0\right\}$ be a right continuous semi-Markovian process on a complete probability space $(\Omega, \mathcal{F}, \mathcal{P})$ taking values in a finite state space $\mathcal{L}=\{1,2, \ldots, s\}$. The evolution of the semi-Markovian process $r_{t}$ is 
governed by the following probability transitions:

$$
\operatorname{Pr}\left\{r_{t+\bar{h}}=j \mid r_{t}=i\right\}= \begin{cases}\pi_{i j}(\bar{h}) \bar{h}+o(\bar{h}), & j \neq i \\ 1+\pi_{i i}(\bar{h}) \bar{h}+o(\bar{h}), & j=i\end{cases}
$$

where $\pi_{i j}(\bar{h})$ is the transition rate from mode $i$ at time $t$ to mode $j$ at time $t+\bar{h}$ when $j \neq i$ and $\pi_{i i}(\bar{h})=$ $-\sum_{j=1, j \neq i}^{s} \pi_{i j}(\bar{h}) ; o(\bar{h})$ is little-o notation defined by $\lim _{\bar{h} \rightarrow 0} o(\bar{h}) / \bar{h}=0$.

Remark 2.1. In practice, the transition rate $\pi_{i j}(\bar{h})$ is general bounded by $\underline{\pi}_{i j} \leq \pi_{i j}(\bar{h}) \leq \bar{\pi}_{i j}$, where $\underline{\pi}_{i j}$, $\bar{\pi}_{i j}$ are real constant scalars. In this case, $\pi_{i j}(\bar{h})$ can always be described by $\pi_{i j}(\bar{h})=\pi_{i j}+\triangle \pi_{i j}$, where $\pi_{i j}=\frac{1}{2}\left(\bar{\pi}_{i j}+\underline{\pi}_{i j}\right)$ and $\left|\triangle \pi_{i j}\right| \leq \tilde{\pi}_{i j}$ with $\tilde{\pi}_{i j}=\frac{1}{2}\left(\bar{\pi}_{i j}-\underline{\pi}_{i j}\right)$.

Consider the following semi-Markovian jump slave system with sampled-data feedback control:

$$
\left\{\begin{array}{l}
\dot{y}(t)=A\left(r_{t}\right) y(t)+B\left(r_{t}\right) y(t-h)+W\left(r_{t}\right) f(D y(t))+u(t), \\
\lambda(t)=C\left(r_{t}\right) y(t), \\
u(t)=K(\gamma(t)-\lambda(t)),
\end{array}\right.
$$

where $y(t) \in \mathbf{R}^{n}$ is the state vector of slave system, $\lambda(t) \in \mathbf{R}^{l}$ is the output of slave system, $u(t)$ is slave system control input, $K$ is the sampled-data feedback control gain matrix to be designed.

The measured outputs $\gamma(t)$ and $\lambda(t)$ are sampled and measured only at each sampling instant $t_{k}$, satisfying $0<t_{1}<t_{2}<\cdots<t_{k}<\cdots$ and $\lim _{k \rightarrow \infty} t_{k}=\infty$. In practical systems, especially in networked environment, packet dropouts could inevitably occur in the sampler-controller channel. Let $t_{l^{(j)}}, j=0,1,2, \ldots$ denote the sampling instants that the packet transmitted successfully to the controller. Clearly, the sequence $\left\{t_{l^{(j)}}\right\}_{j=0}^{\infty}$ is a subsequence of sequence $\left\{t_{k}\right\}_{k=0}^{\infty}$. Therefore, the controller takes the following form:

$$
u(t)=K\left(\gamma\left(t_{l^{(j)}}\right)-\lambda\left(t_{l^{(j)}}\right)\right), \quad t_{l^{(j)}} \leq t<t_{l^{(j+1)}}, j=0,1,2, \ldots
$$

By defining the error signal as $e(t)=x(t)-y(t)$, the error system can be represented as follows:

$$
\dot{e}(t)=A\left(r_{t}\right) e(t)+B\left(r_{t}\right) e(t-h)+W\left(r_{t}\right) g(t)-K C\left(r_{t}\right) e\left(t_{l^{(j)}}\right), \quad t_{l^{(j)}} \leq t<t_{l^{(j+1)}}, j=0,1,2, \ldots
$$

where $g(t)=f(D e(t)+D y(t))-f(D y(t))$. Let $D=\left[d_{1}, d_{2}, \ldots, d_{m_{f}}\right]^{T}$ with $d_{s} \in \mathbf{R}^{n}, s=1,2, \ldots, m_{f}$. It can be found that the functions $g_{s}(\cdot)$ satisfy the following condition:

$$
k_{s}^{-} \leq \frac{g_{s}}{d_{s}^{T} e}=\frac{f_{s}\left(d_{s}^{T}(e+y)\right)-f_{s}\left(d_{s}^{T} y\right)}{d_{s}^{T} e} \leq k_{s}^{+}, \quad s=1,2, \ldots, m_{f}
$$

where $d_{s}^{T} e \neq 0$.

Assumption 2.2. At the sampling instant $t_{l^{(j+1)}}$, the number of successive packet dropouts since the last sampling instant $t_{l^{(j)}}$ is denoted as $n_{j, j+1}$. The variable $n_{j, j+1}$ takes values in $\bar{\zeta}_{n_{j, j+1}}=\{0,1,2, \ldots, \bar{N}\}$ arbitrarily, where $\bar{N}=\max _{j \in N}\left\{n_{j, j+1}\right\}$. 
To design the state-feedback controller, the concept of the input-delay approach has been employed, that is

$$
u(t)=K C\left(r_{t}\right) e(t-\rho(t)), \quad t_{l^{(j)}} \leq t<t_{l^{(j+1)}}, j=0,1,2, \ldots
$$

where $\rho(t)=t-t_{l^{(j)}}$. For any given $l^{(j)}, j=0,1,2, \ldots$, there exists $k \in \mathbf{N}$ such that $t_{l^{(j)}}=t_{k}$. Let $t_{k+1}-t_{k}=T_{k+1}$, then, the distance between two adjacent sampling instants that the packet transmitted successfully to the controller

$$
t_{l^{(j+1)}}-t_{l^{(j)}}=T_{k+1}+T_{k+2}+\cdots+T_{k+n_{j, j+1}+1}
$$

where $T_{k+n_{j, j+1+1}}\left(0 \leq n_{j, j+1} \leq \bar{N}\right)$ represents the sampling period. According to the definition of $\rho(t)$, we obtain $0 \leq \rho(t)<t_{l^{(j+1)}}-t_{l^{(j)}}, t_{l^{(j)}} \leq t<t_{l^{(j+1)}}$. Then, it yields

$$
0 \leq \rho(t)<T_{k+1}+T_{k+2}+\cdots+T_{k+n_{j, j+1}+1}\left(0 \leq n_{j, j+1} \leq \bar{N}\right)
$$

Then, the control input can be written as

$$
u(t)=K C\left(r_{t}\right) e(t-\rho(t))
$$

with $\rho(t) \in\left[0, T_{k+1}+T_{k+2}+\cdots+T_{k+n_{j, j+1}+1}\right)$.

Assumption 2.3. $T_{k+n_{j, j+1}+1}\left(0 \leq n_{j, j+1} \leq \bar{N}\right)$ takes value in $\mathcal{Z}_{T}=\left\{\rho_{1}, \rho_{2}, \ldots, \rho_{m}\right\}$ with $\rho_{1}<\rho_{2}<\ldots<$ $\rho_{m}$. For the sake of simplicity, the probability of the occurrence of each is known, that is

$$
\operatorname{Prob}\left\{T_{k+n_{j, j+1}+1}=\rho_{i}\right\}=\beta_{i}, \quad i=1,2, \ldots, m,
$$

where $\beta_{i} \in[0,1]$ and $\sum_{i=1}^{m} \beta_{i}=1$.

Remark 2.2. Not only the variable $n_{j, j+1}$ is stochastic, but also the sampling period $T_{k+n_{j, j+1}+1}(0 \leq$ $\left.n_{j, j+1} \leq \bar{N}\right)$ takes values in $\mathcal{Z}_{T}$ randomly. That is, the upper bound of the stochastic delay $\rho(t)$ is subject to double randomness.

Notice that $\rho(t)$ is an interval stochastic delay taking values in $\left[0,(\bar{N}+1) \rho_{m}\right)$, without loss of generality, we divide $\left[0,(\bar{N}+1) \rho_{m}\right)$ into $m$ subintervals. Compared with enlarging the upper bound $\tilde{T}_{k+1+n_{j, j+1}}=$ $T_{k+1}+T_{k+2}+\cdots+T_{k+n_{j, j+1}+1}$ of $\rho(t)$ to $\left[0,(\bar{N}+1) \tau_{m}\right)$, in the following, by recurring to the celebrated formula of total probability, we will explicitly obtain the probability distribution values of the stochastic delay $\rho(t)$ taking values in those $m$ subintervals.

Assumption 2.4. The variables $n_{j, j+1}$ and $T_{k+n_{j, j+1}+1}$ are mutually independent. 
Consider the occurrence probabilities of $\mathrm{m}$ sampling periods taking value in $\mathcal{Z}_{T}$ to be independent. When $n_{j, j+1}=0$, the conditional probability distribution of $\rho(t)$ is calculated as:

$$
\begin{aligned}
& \operatorname{Prob}\left\{\rho_{i-1} \leq \rho(t)<\rho_{i} \mid n_{j, j+1}=0\right\} \\
= & \sum_{l_{1}=1}^{m} \operatorname{Prob}\left\{\rho_{i-1} \leq \rho(t)<\rho_{i} \mid n_{j, j+1}=0, T_{k+1}=\rho_{l_{1}}\right\} \times \operatorname{Prob}\left\{T_{k+1}=\rho_{l_{1}}\right\} \\
= & \frac{\rho_{i}-\rho_{i-1}}{\rho_{i}} \beta_{i}+\frac{\rho_{i}-\rho_{i-1}}{\rho_{i+1}} \beta_{i+1}+\ldots+\frac{\rho_{i}-\rho_{i-1}}{\rho_{m}} \beta_{m} \\
= & \sum_{p_{1}+p_{2}+\ldots+p_{i-1}=0, p_{i}+p_{i+1}+\ldots+p_{m}=1} \frac{\rho_{i}-\rho_{i-1}}{p_{1} \rho_{1}+p_{2} \rho_{2}+\ldots+p_{m} \rho_{m}} \beta_{1}{ }^{p_{1}} \beta_{2}{ }^{p_{2}} \ldots \beta_{m}{ }^{p_{m}} \\
= & \bar{\beta}_{0 i}, \quad i=1,2, \ldots, m,
\end{aligned}
$$

where $p_{i}(i=1,2, \ldots, m)$ is a non-negative integer.

When $n_{j, j+1}=v(v=1,2, \ldots, \bar{N})$, the conditional probability distribution of $\rho(t)$ is calculated as:

$$
\begin{aligned}
& \operatorname{Prob}\left\{\rho_{i-1} \leq \rho(t)<\rho_{i} \mid n_{j, j+1}=v\right\} \\
& =\sum_{l_{1}, l_{2}, \ldots, l_{v+1}=1}^{m} \operatorname{Prob}\left\{\rho_{i-1} \leq \rho(t)<\rho_{i} \mid n_{j, j+1}=v, T_{k+1}=\rho_{l_{1}}, \ldots, T_{k+v+1}=\rho_{l_{v+1}}\right\} \\
& \times \operatorname{Prob}\left\{T_{k+1}=\rho_{l_{1}}\right\} \times \operatorname{Prob}\left\{T_{k+2}=\rho_{l_{2}}\right\} \times \ldots \times \operatorname{Prob}\left\{T_{k+v+1}=\rho_{l_{v+1}}\right\} \\
& =\sum_{p_{1}+p_{2}+\ldots+p_{m}=v+1} \frac{\rho_{i}-\rho_{i-1}}{p_{1} \rho_{1}+p_{2} \rho_{2}+\ldots+p_{m} \rho_{m}} C_{v+1}^{p_{1}} \beta_{1}{ }^{p_{1}} C_{v+1-p_{1}}^{p_{2}} \beta_{2}{ }^{p_{2}} \ldots C_{p_{m-1}+p_{m}}^{p_{m-1}} \beta_{m-1}{ }^{p_{m-1}} C_{p_{m}}^{p_{m}} \beta_{m}{ }^{p_{m}} \\
& =\bar{\beta}_{v i}, i=1,2, \ldots, m-1 \text {; } \\
& \operatorname{Prob}\left\{\rho_{m-1} \leq \rho(t)<\left(n_{j, j+1}+1\right) \rho_{m} \mid n_{j, j+1}=v\right\} \\
& =\sum_{p_{1}+p_{2}+\ldots+p_{m}=v+1}\left(1-\frac{\rho_{m-1}}{p_{1} \rho_{1}+p_{2} \rho_{2}+\ldots+p_{m} \rho_{m}}\right) C_{v+1}^{p_{1}} \beta_{1}{ }^{p_{1}} C_{v+1-p_{1}}^{p_{2}} \beta_{2}{ }^{p_{2}} \ldots C_{p_{m-1}+p_{m}}^{p_{m-1}} \beta_{m-1}{ }^{p_{m-1}} C_{p_{m}}^{p_{m}} \beta_{m}{ }^{p_{m}} \\
& =\bar{\beta}_{v m} \text {, }
\end{aligned}
$$

where $\rho_{0}=0$ and $\bar{\beta}_{i 1}+\bar{\beta}_{i 2}+\cdots+\bar{\beta}_{i m}=1, i=0,1,2, \cdots, \bar{N}$.

Suppose that the packet loss rate is $\bar{\theta}$, then $\operatorname{Prob}\left\{n_{j, j+1}=v\right\}=(1-\bar{\theta}) \bar{\theta}^{v}, v=0,1,2, \ldots, \bar{N}-1 ; \operatorname{Prob}\left\{n_{j, j+1}=\right.$ $\bar{N}\}=\bar{\theta}^{\bar{N}}$. From the discussion above, the probability distribution values of $\rho(t)$ in $\left[0,(\bar{N}+1) \rho_{m}\right)$ can be calculated as:

$$
\begin{aligned}
& \operatorname{Prob}\left\{\rho_{i-1} \leq \rho(t)<\lambda_{i} \rho_{i}\right\}=\sum_{v=0}^{\bar{N}-1} \bar{\beta}_{v i}(1-\bar{\theta}) \bar{\theta}^{v}+\bar{\beta}_{\bar{N} i} \bar{\theta}^{\bar{N}}=\theta_{i}, i=1,2, \ldots, m-1 \\
& \operatorname{Prob}\left\{\rho_{m-1} \leq \rho(t)<\lambda_{m} \rho_{m}\right\}=\sum_{v=0}^{\bar{N}-1} \bar{\beta}_{v m}(1-\bar{\theta}) \bar{\theta}^{v}+\bar{\beta}_{\bar{N} m} \bar{\theta}^{\bar{N}}=\theta_{m}
\end{aligned}
$$

where $\lambda_{i}=1(i=1,2, \cdots, m-1), \lambda_{m}=\bar{N}+1$ and $\theta_{1}+\theta_{2}+\cdots+\theta_{m}=1$.

Introduce the new indicator functions

$$
\alpha_{i}(t)= \begin{cases}1 & \bar{\rho}_{i}(t)=\rho(t) \in\left[\rho_{i-1}, \lambda_{i} \rho_{i}\right), \quad i=1,2, \ldots, m \\ 0 & \text { otherwise }\end{cases}
$$


Then, we have $E\left\{\alpha_{i}(t)\right\}=\operatorname{Prob}\left\{\rho(t) \in\left[\rho_{i-1}, \lambda_{i} \rho_{i}\right)\right\}=\theta_{i}, i=1,2, \ldots, m$. Controller with $m$ sampling intervals can be converted into

$$
u(t)=\sum_{i=1}^{m} \alpha_{i}(t) K C\left(r_{t}\right) e\left(t-\bar{\rho}_{i}(t)\right), \quad t \in\left[t_{l^{(j)}}, t_{l^{(j+1)}}\right),
$$

with $\rho_{i-1} \leq \bar{\rho}_{i}(t)<\lambda_{i} \rho_{i}, i=1,2, \ldots, m$. Therefore, the error system (6) with $m$ sampling intervals can be rewritten as follow:

$$
\dot{e}(t)=A\left(r_{t}\right) e(t)+B\left(r_{t}\right) e(t-h)+W\left(r_{t}\right) g(t)-\sum_{l=1}^{m} \alpha_{l}(t) K C\left(r_{t}\right) e\left(t-\bar{\rho}_{l}(t)\right)
$$

for $t \in\left[t_{l^{(j)}}, t_{l^{(j+1)}}\right), j=0,1,2, \ldots$, where $\rho_{l-1} \leq \bar{\rho}_{l}(t)<\lambda_{l} \rho_{l}, l=1,2, \ldots, m$.

Lemma 2.1. ([35]) For a positive definite matrix $S$ and any differentiable function $x$ on $[a, b] \longrightarrow \boldsymbol{R}^{n}$, the following inequality holds:

$$
-\int_{a}^{b} \dot{x}^{T}(s) S \dot{x}(s) d s \leq \frac{1}{a-b} \bar{x}^{T}(s) \bar{S} \bar{x}(s) d s
$$

where

$$
\bar{x}(a, b)=\left[\begin{array}{c}
x(b) \\
x(a) \\
\frac{1}{b-a} \int_{a}^{b} x(s) d s
\end{array}\right]^{T}, \quad \bar{S}=\left[\begin{array}{ccc}
S & -S & 0 \\
* & S & 0 \\
* & * & 0
\end{array}\right]+\frac{\pi^{2}}{4}\left[\begin{array}{ccc}
S & S & -2 S \\
* & S & -2 S \\
* & * & 4 S
\end{array}\right] .
$$

Lemma 2.2. ([7]) For a given matrix $M>0$, given scalars $a$ and $b$ satisfying $a<b$, the following inequality holds for all continuously differentiable function $r$ in $[a, b] \longrightarrow \boldsymbol{R}^{n}$ :

$$
-\frac{(b-a)^{2}}{2} \int_{a}^{b} \int_{\theta}^{b} r^{T}(s) M r(s) d s d \theta \leq-\int_{a}^{b} \int_{\theta}^{b} r^{T}(s) d s d \theta M \int_{a}^{b} \int_{\theta}^{b} r(s) d s d \theta-2 \Theta^{T} M \Theta
$$

where $\Theta=-\int_{a}^{b} \int_{\theta}^{b} r(s) d s d \theta+\frac{3}{b-a} \int_{a}^{b} \int_{\lambda}^{b} \int_{\theta}^{b} r(s) d s d \theta d \lambda$.

Lemma 2.3. ([7]) For a given symmetric positive definite matrix $R$, arbitrary scalars $0 \leq x_{1} \leq 1$ and $0 \leq x_{2} \leq 1$ $\left(x_{1}+x_{2}=1\right)$, and for differentiable signal $r(t)$ in $\left[0, x_{3}\right] \longrightarrow \boldsymbol{R}^{n}$, the following inequality holds:

$$
-\int_{t-h}^{t} \dot{r}^{T}(s) R \dot{r}(s) d s \leq-\left[\begin{array}{c}
r(t) \\
r(t-h) \\
\int_{t-h}^{t} r(s) d s
\end{array}\right]^{T} \Omega_{1}\left(x_{1}, x_{2}, x_{3}\right) \otimes R\left[\begin{array}{c}
r(t) \\
r(t-h) \\
\int_{t-h}^{t} r(s) d s
\end{array}\right],
$$

where

$$
\Omega_{1}\left(x_{1}, x_{2}, x_{3}\right)=\left[\begin{array}{ccc}
\frac{4 x_{2}}{x_{3}} & \frac{2 x_{2}}{x_{3}} & \frac{-6 x_{2}}{x_{3}^{2}} \\
\frac{2 x_{2}}{x_{3}} & \frac{4-2 x_{1}}{x_{3}} & \frac{-6+4 x_{1}}{x_{3}^{2}} \\
\frac{-6 x_{2}}{x_{3}^{2}} & \frac{-6+4 x_{1}}{x_{3}^{2}} & \frac{12-10 x_{1}}{x_{3}^{3}}
\end{array}\right] .
$$

Lemma 2.4. ([38]) Given any scalar $\varepsilon$ and square matrix $Q \in \boldsymbol{R}^{n \times n}$, the following inequality

$$
\varepsilon\left(Q+Q^{T}\right) \leq \varepsilon^{2} T+Q T^{-1} Q^{T}
$$

holds for any symmetric positive definite matrix $T \in \boldsymbol{R}^{n \times n}$. 
Definition 2.1. Master system (1) and slave system (4) are said to be stochastically synchronous if error system (12) is stochastically stable, that is, for any initial condition $e(t)=\phi(t)$ defined on the interval $\left[t_{l^{(0)}}-\max \left\{(\bar{N}+1) \rho_{m}, h\right\}\right.$, $\left.t_{l(0)}\right]$, the following condition is satisfied:

$$
\lim _{T \rightarrow \infty} E\left\{\int_{t_{l}(0)}^{T}|| e(s) \|^{2} d s \mid \phi(t)\right\}<\infty .
$$

\section{Main results}

In this section, the sufficient conditions for the error system (12) to be stochastically stable are established. For the sake of simplicity of matrix representation, the notations for some vectors and matrices are defined in Appendix.

Theorem 3.1. For given scalars $h>0,0<\bar{\alpha}<1, \delta, 0 \leq \mu_{i_{1}} \leq 1\left(i_{1}=1,2,3,4\right), \mu_{1}+\mu_{2}=1, \mu_{3}+\mu_{4}=$ 1 , the master system (1) and the slave system (4) are stochastically synchronous if there exist matrices $P_{q}>0$, $R_{i_{2}}>0\left(i_{2}=1,2,3,4,5,6,7\right), Q_{i_{3}}>0, S_{1 i_{3}}>0, S_{2 i_{3}}>0\left(i_{3}=1,2, \ldots, m\right), L_{1}=\operatorname{diag}\left\{\omega_{1}, \omega_{2}, \ldots, \omega_{m}\right\}>0$, $L_{2}=\operatorname{diag}\left\{\iota_{1}, \iota_{2}, \ldots, \iota_{m}\right\}>0, V_{j l}=\operatorname{diag}\left\{v_{j 1 l}, v_{j 2 l}, \ldots, v_{j m_{f}} l\right\}>0(l=1,2 ; j=1,2,3)$, and any appropriately dimensioned matrices $\bar{W}_{i_{4}}\left(i_{4}=1,2, \ldots, m\right), H_{1}, H_{2}, N, U, M=\left[M_{1}, M_{2}, M_{3}\right]$ and $\bar{M}=[M, 0]$, such that the following matrix inequalities hold for all $q \in \mathcal{L}$ :

$$
\begin{aligned}
& \begin{array}{l}
\Lambda_{q}\left((\bar{N}+1) \rho_{m}\right)=\left[\begin{array}{c}
P_{q}+2 \delta((\bar{N}+1) \\
*
\end{array}\right. \\
{\left[\begin{array}{cc}
S_{1 i} & \bar{W}_{i}^{T} \\
* & S_{1 i}
\end{array}\right]>0, \quad i=1,2, \ldots, m,}
\end{array} \\
& \Gamma_{1}(\hat{T})=\Upsilon_{0}+\Upsilon_{01}+\Upsilon_{1}(\hat{T})<0, \\
& \hat{\Gamma}_{2}(\hat{T})=\left[\begin{array}{cc}
\Upsilon_{0}+\Upsilon_{02} & \hat{T}^{\frac{1}{2}} \bar{M}^{T} \\
* & -R_{1}
\end{array}\right]<0, \quad \hat{T}=0,(\bar{N}+1) \rho_{m},
\end{aligned}
$$

where $\Upsilon_{0}, \Upsilon_{01}, \Upsilon_{02}$ and $\Upsilon_{1}(\hat{T})$ are defined in Appendix.

Moreover, the gain matrix is given by

$$
K=N^{-1} U
$$

Proof. Consider the following Lyapunov functional candidate for the error system $(12)\left(t \in\left[t_{l^{(j)}}, t_{l^{(j+1)}}\right)\right)$ :

$$
V(t)=\sum_{i=1}^{7} V_{i}(t)
$$

where

$$
\begin{aligned}
& V_{1}(t)=e^{T}(t) P\left(r_{t}\right) e(t)+\left(t_{l^{(j+1)}}-t\right) \varepsilon^{T}(t) \mathcal{H} \varepsilon(t), \\
& V_{2}(t)=\left(t_{l^{(j+1)}}-t\right) \int_{t_{l(j)}}^{t} \dot{e}^{T}(s) R_{1} \dot{e}(s) d s \\
& V_{3}(t)=\int_{t-\bar{\alpha} h}^{t} e^{T}(s) R_{2} e(s) d s+\int_{t-h}^{t-\bar{\alpha} h} e^{T}(s) R_{3} e(s) d s, \\
& V_{4}(t)=\int_{-\bar{\alpha} h}^{0} \int_{t+\theta}^{t} \dot{e}^{T}(s) R_{4} \dot{e}(s) d s d \theta+\int_{-h}^{-\bar{\alpha} h} \int_{t+\theta}^{t} \dot{e}^{T}(s) R_{5} \dot{e}(s) d s d \theta,
\end{aligned}
$$




$$
\begin{aligned}
& V_{5}(t)=\frac{\bar{\alpha}^{2}}{2} \int_{t-\bar{\alpha} h}^{t} \int_{\lambda}^{t} \int_{\theta}^{t} \dot{e}^{T}(s) R_{6} \dot{e}(s) d s d \theta d \lambda+\frac{1}{2} \int_{t-h}^{t} \int_{\lambda}^{t} \int_{\theta}^{t} \dot{e}^{T}(s) R_{7} \dot{e}(s) d s d \theta d \lambda, \\
& V_{6}(t)=2 \sum_{s=1}^{m_{f}} \omega_{s} \int_{0}^{d s^{T} e(t)}\left[g_{s}(\theta)-k_{s}^{-} \theta\right] d \theta+2 \sum_{s=1}^{m_{f}} \iota_{s} \int_{0}^{d s^{T} e(t)}\left[k_{s}^{+} \theta-g_{s}(\theta)\right] d \theta \\
& V_{7}(t)=\sum_{i=1}^{m} \theta_{i} \int_{t-\lambda_{i} \rho_{i}}^{t-\rho_{i-1}} e^{T}(s) Q_{i} e(s) d s+\sum_{i=1}^{m} \theta_{i} \bar{\rho}_{i} \int_{-\lambda_{i} \rho_{i}}^{-\rho_{i-1}} \int_{t+\theta}^{t} \dot{e}^{T}(s) Z_{i} \dot{e}(s) d s d \theta
\end{aligned}
$$

with $\bar{\rho}_{i}=\lambda_{i} \rho_{i}-\rho_{i-1}, Z_{i}=S_{1 i}+S_{2 i}, i=1,2, \ldots, m$.

According to the assumptions, we know that $V_{2}(t), V_{3}(t), V_{4}(t), V_{5}(t), V_{6}(t)$ and $V_{7}(t)$ are positive. If $V_{1}(t)$ is positive definite, we can obtain that $V(t)$ is positive definite. For $\forall r_{t}=q \in \mathcal{L}$, we can get

$$
\begin{aligned}
V_{1}(t) & =e^{T}(t) P_{q} e(t)+\left(t_{l^{(j+1)}}-t\right) \varepsilon^{T}(t) \mathcal{H} \varepsilon(t) \\
& =\left[\begin{array}{c}
e(t) \\
e\left(t_{l^{(j)}}\right)
\end{array}\right]^{T}\left(\left[\begin{array}{rr}
P_{q} & 0 \\
* & 0
\end{array}\right]+\left(t_{l^{(j+1)}}-t\right) \mathcal{H}\right)\left[\begin{array}{c}
e(t) \\
e\left(t_{l^{(j)}}\right)
\end{array}\right] \\
& =\left[\begin{array}{c}
e(t) \\
e\left(t_{l^{(j)}}\right)
\end{array}\right]^{T}\left(\frac{t-t_{l^{(j)}}}{\tilde{T}_{k+1+n_{j, j+1}}} \Lambda_{q}(0)+\frac{t_{l^{(j+1)}}-t}{\tilde{T}_{k+1+n_{j, j+1}}} \Lambda_{q}\left(\tilde{T}_{k+1+n_{j, j+1}}\right)\right)\left[\begin{array}{c}
e(t) \\
e\left(t_{l^{(j)}}\right)
\end{array}\right],
\end{aligned}
$$

and

$$
\Lambda_{q}\left(\tilde{T}_{k+1+n_{j, j+1}}\right)=\frac{\tilde{T}_{k+1+n_{j, j+1}}}{(\bar{N}+1) \rho_{m}} \Lambda_{q}\left((\bar{N}+1) \rho_{m}\right)+\frac{(\bar{N}+1) \rho_{m}-\tilde{T}_{k+1+n_{j, j+1}}}{(\bar{N}+1) \rho_{m}} \Lambda_{q}(0) .
$$

From (13) and $P_{q}>0$, it is clear that $V(t)>V_{1}(t)$. It is noted that, two $\left(t_{l^{(j)}}, t_{l^{(j+1)}}\right)$-dependent terms $\left(t_{l^{(j+1)}}-t\right) \varepsilon^{T}(t) \mathcal{H} \varepsilon(t)$ and $V_{2}(t)$ are introduced in (17), which make full use of the information available on the actual sampling pattern. In addition, $V(t)$ is continuous on the whole interval $[0, \infty]$ because $\left(t_{l^{(j)}}, t_{l^{(j+1)}}\right)$-dependent terms in $V_{1}(t)$ and $V_{2}(t)$ vanish before and after the jump $t_{l^{(j)}}$.

Calculating the time derivative of $V(t)$ along the trajectory of error system (12), and taking the mathematical expectation, we have

$$
\begin{aligned}
E\left\{\dot{V}_{1}(t)\right\} & =\lim _{\triangle \rightarrow 0} E\left\{\frac{V_{1}(t+\triangle)-V_{1}(t)}{\triangle}\right\} \\
& =\lim _{\triangle \rightarrow 0} \frac{1}{\triangle} E\left\{\sum_{j=1, j \neq q}^{s} \operatorname{Pr}\left\{r_{t+\triangle}=j \mid r_{t}=q\right\} e^{T}(t+\triangle) P_{j} e(t+\triangle)\right. \\
& \left.+\operatorname{Pr}\left\{r_{t+\triangle}=q \mid r_{t}=q\right\} e^{T}(t+\triangle) P_{q} e(t+\triangle)-e^{T}(t) P_{q} e(t)\right\}-\varepsilon^{T}(t) \mathcal{H} \varepsilon(t)+2\left(t_{l}(j+1)-t\right) \varepsilon^{T}(t) \mathcal{H} \dot{\varepsilon}(t) \\
& =\lim _{\triangle \rightarrow 0} \frac{1}{\triangle} E\left\{\sum_{j=1, j \neq q}^{s} \frac{\varrho_{q j}\left(G_{q}(\bar{h}+\triangle)-G_{q}(\bar{h})\right)}{1-G_{q}(\bar{h})} e^{T}(t+\triangle) P_{j} e(t+\triangle)\right. \\
& \left.+\frac{1-G_{q}(\bar{h}+\triangle)}{1-G_{q}(\bar{h})} e^{T}(t+\triangle) P_{q} e(t+\triangle)-e^{T}(t) P_{q} e(t)\right\}-\varepsilon^{T}(t) \mathcal{H} \varepsilon(t)+2\left(t_{l(j+1)}-t\right) \varepsilon^{T}(t) \mathcal{H} \dot{\varepsilon}(t)
\end{aligned}
$$

where $\bar{h}$ is the time elapsed when the system stays at mode $p$ from the last jump; $G_{q}(t)$ is the cumulative distribution function of the sojourn time when the system remains in mode $p$, and $\varrho_{q j}$ is the probability intensity of the system jump from mode $p$ to mode $j$. Given that $\triangle$ is small, the first order approximation of $e(t+\triangle)$ is

$$
\begin{aligned}
e(t+\triangle) & =e(t)+\dot{e}(t) \triangle+o(\triangle) \\
& =\left(I+A_{q} \triangle\right) e(t)+\left(B_{q} e(t-h)+W_{q} g(t)-\sum_{l=1}^{m} \alpha_{l}(t) K C_{q} e\left(t-\bar{\rho}_{l}(t)\right)\right) \triangle+o(\triangle) .
\end{aligned}
$$


Then,

$$
\begin{aligned}
E\left\{\dot{V}_{1}(t)\right\} & =\lim _{\triangle \rightarrow 0} \frac{1}{\triangle}\left\{\sum _ { j = 1 , j \neq q } ^ { s } \frac { \varrho _ { q j } ( G _ { q } ( \overline { h } + \triangle ) - G _ { q } ( \overline { h } ) ) } { 1 - G _ { q } ( \overline { h } ) } \left(\left[\left(I+A_{q} \triangle\right) e(t)\right]^{T} P_{j}\left[\left(I+A_{q} \triangle\right) e(t)\right]\right.\right. \\
& +2\left[\left(I+A_{q} \triangle\right) e(t)\right]^{T} P_{j}\left[\left(B_{q} e(t-h)+W_{q} g(t)\right) \triangle\right] \\
& -2 \sum_{l=1}^{m} \theta_{l}\left[\left(I+A_{q} \triangle\right) e(t)\right]^{T} P_{j}\left[K C_{q} e\left(t-\bar{\rho}_{l}(t)\right) \triangle\right] \\
& +\left[\left(B_{q} e(t-h)+W_{q} g(t)\right) \triangle\right]^{T} P_{j}\left[\left(B_{q} e(t-h)+W_{q} g(t)\right) \triangle\right] \\
& -2 \sum_{l=1}^{m} \theta_{l}\left[\left(B_{q} e(t-h)+W_{q} g(t)\right) \triangle\right]^{T} P_{j}\left[K C_{q} e\left(t-\bar{\rho}_{l}(t)\right) \triangle\right] \\
& \left.+\sum_{l=1}^{m} \theta_{l}\left[K C_{q} e\left(t-\bar{\rho}_{l}(t)\right) \triangle\right]^{T} P_{j}\left[K C_{q} e\left(t-\bar{\rho}_{l}(t)\right) \triangle\right]+o(\triangle)\right) \\
& +\frac{1-G_{q}(\bar{h}+\triangle)}{1-G_{q}(\bar{h})}\left(\left[\left(I+A_{q} \triangle\right) e(t)\right]^{T} P_{q}\left[\left(I+A_{q} \triangle\right) e(t)\right]\right. \\
& +2\left[\left(I+A_{q} \triangle\right) e(t)\right]^{T} P_{q}\left[\left(B_{q} e(t-h)+W_{q} g(t)\right) \triangle\right] \\
& -2 \sum_{l=1}^{m} \theta_{l}\left[\left(I+A_{q} \triangle\right) e(t)\right]^{T} P_{q}\left[K C_{q} e\left(t-\bar{\rho}_{l}(t)\right) \triangle\right] \\
& +\left[\left(B_{q} e(t-h)+W_{q} g(t)\right) \triangle\right]^{T} P_{q}\left[\left(B_{q} e(t-h)+W_{q} g(t)\right) \triangle\right] \\
& +2 \sum_{l=1}^{m} \theta_{l}\left[\left(B_{q} e(t-h)+W_{q} g(t)\right) \triangle\right]^{T} P_{q}\left[K C_{q} e\left(t-\bar{\rho}_{l}(t)\right) \triangle\right] \\
& +\sum_{l=1}^{m} \theta_{l}\left[K C_{q} e\left(t-\bar{\rho}_{l}(t)\right) \triangle\right]^{T} P_{q}\left[K C_{q} e\left(t-\bar{\rho}_{l}(t)\right) \triangle\right] \\
& \left.o(\triangle) e^{T}(t) P_{q} e(t)\right\}-\varepsilon^{T}(t) \mathcal{H} \varepsilon(t)+2\left(t_{l}(j+1)-t\right) \varepsilon^{T}(t) \mathcal{H} \dot{\varepsilon}(t) . \\
& \\
&
\end{aligned}
$$

By the same techniques used in [38], we have

$$
\lim _{\triangle \rightarrow 0} \frac{G_{q}(\bar{h}+\triangle)-G_{q}(\bar{h})}{\triangle\left(1-G_{q}(\bar{h})\right)}=\pi_{q}(\bar{h}), \quad \lim _{\triangle \rightarrow 0} \frac{1-G_{q}(\bar{h}+\triangle)}{1-G_{q}(\bar{h})}=1, \quad \lim _{\triangle \rightarrow 0} \frac{G_{q}(\bar{h}+\triangle)-G_{q}(\bar{h})}{1-G_{q}(\bar{h})}=0 .
$$

where $\pi_{q}(\bar{h})$ is the transition rate of the system jumping from mode $q$.

Define

$$
\pi_{q j}(\bar{h})=\varrho_{q j} \pi_{q}(\bar{h}), j \neq q, \quad \pi_{q q}(\bar{h})=-\sum_{j=1, j \neq q}^{s} \pi_{q j}(\bar{h}) .
$$

Then,

$$
\begin{aligned}
E\left\{\dot{V}_{1}(t)\right\} & =2 e^{T}(t) P_{q} \dot{e}(t)+\sum_{j=1}^{s} \pi_{q j}(h) e^{T}(t) P_{j} e(t)-\varepsilon^{T}(t) \mathcal{H} \varepsilon(t)+2\left(t_{l(j+1)}-t\right) \varepsilon^{T}(t) \mathcal{H} \dot{\varepsilon}(t) \\
& =\xi^{T}(t)\left\{\frac{t_{l(j+1)}-t}{\tilde{T}_{k+1+n_{j, j+1}}}\left(2 \tilde{e}_{1} P_{q} \tilde{e}_{2}^{T}+\sum_{j=1}^{s} \pi_{q j}(h) \tilde{e}_{1} P_{j} \tilde{e}_{1}^{T}-\left[\tilde{e}_{1}, \tilde{e}_{3}\right] \mathcal{H}\left[\tilde{e}_{1}, \tilde{e}_{3}\right]^{T}+2 \tilde{T}_{k+1+n_{j, j+1}}\left[\tilde{e}_{1}, \tilde{e}_{3}\right] \mathcal{H}\left[\tilde{e}_{2}, 0\right]^{T}\right)\right. \\
& \left.+\frac{t-t_{l(j)}}{\tilde{T}_{k+1+n_{j, j+1}}}\left(2 \tilde{e}_{1} P_{q} \tilde{e}_{2}^{T}+\sum_{j=1}^{s} \pi_{q j}(h) \tilde{e}_{1} P_{j} \tilde{e}_{1}^{T}-\left[\tilde{e}_{1}, \tilde{e}_{3}\right] \mathcal{H}\left[\tilde{e}_{1}, \tilde{e}_{3}\right]^{T}\right)\right\} \xi(t),
\end{aligned}
$$




$$
E\left\{\dot{V}_{2}(t)\right\}=-\int_{t_{l}(j)}^{t} \dot{e}^{T}(s) R_{1} \dot{e}(s) d s+\left(t_{l^{(j+1)}}-t\right) \dot{e}^{T}(t) R_{1} \dot{e}(t) .
$$

Moreover, for an appropriately dimensioned matrix $M=\left[M_{1}, M_{2}, M_{3}\right]$, we can get the following inequality:

$$
\int_{t_{l}(j)}^{t}\left[\begin{array}{c}
\varphi(t) \\
\dot{e}(s)
\end{array}\right]^{T}\left[\begin{array}{cc}
M^{T} R_{1}^{-1} M & M^{T} \\
* & R_{1}
\end{array}\right]\left[\begin{array}{c}
\varphi(t) \\
\dot{e}(s)
\end{array}\right] d s \geq 0 .
$$

This implies,

$$
\begin{aligned}
E\left\{\dot{V}_{2}(t)\right\} & \leq\left(t_{l^{(j+1)}}-t\right) \dot{e}^{T}(t) R_{1} \dot{e}(t)+\left(t-t_{l^{(j)}}\right) \varphi^{T}(t) M^{T} R_{1}^{-1} M \varphi(t)+2 \varphi^{T}(t) M^{T}\left[e(t)-e\left(t_{l^{(j)}}\right)\right] \\
& =\xi^{T}(t)\left\{\frac{t_{l^{(j+1)}}-t}{\tilde{T}_{k+1+n_{j, j+1}}}\left(\tilde{T}_{k+1+n_{j, j+1}} \tilde{e}_{2} R_{1} \tilde{e}_{2}^{T}+2\left[\tilde{e}_{1}, \tilde{e}_{2}, \tilde{e}_{3}\right] M^{T}\left[\tilde{e}_{1}-\tilde{e}_{3}\right]^{T}\right)\right. \\
& \left.+\frac{t-t_{l^{(j)}}}{\tilde{T}_{k+1+n_{j, j+1}}}\left(\tilde{T}_{k+1+n_{j, j+1}}\left[\tilde{e}_{1}, \tilde{e}_{2}, \tilde{e}_{3}\right] M^{T} R_{1}^{-1} M\left[\tilde{e}_{1}, \tilde{e}_{2}, \tilde{e}_{3}\right]^{T}+2\left[\tilde{e}_{1}, \tilde{e}_{2}, \tilde{e}_{3}\right] M^{T}\left[\tilde{e}_{1}-\tilde{e}_{3}\right]^{T}\right)\right\} \xi(t) . \\
E\left\{\dot{V}_{3}(t)\right\} & =e^{T}(t) R_{2} e(t)+e^{T}(t-\bar{\alpha} h)\left(R_{3}-R_{2}\right) e(t-\bar{\alpha} h)-e^{T}(t-h) R_{3} e(t-h) \\
& =\xi^{T}(t)\left\{\frac{t_{l^{(j+1)}}-t}{\tilde{T}_{k+1+n_{j, j+1}}}\left(\tilde{e}_{1} R_{2} \tilde{e}_{1}^{T}+\tilde{e}_{4}\left(R_{3}-R_{2}\right) \tilde{e}_{4}^{T}-\tilde{e}_{5} R_{3} \tilde{e}_{5}^{T}\right)\right. \\
& \left.+\frac{t-t_{l^{(j)}}}{\tilde{T}_{k+1+n_{j, j+1}}}\left(\tilde{e}_{1} R_{2} \tilde{e}_{1}^{T}+\tilde{e}_{4}\left(R_{3}-R_{2}\right) \tilde{e}_{4}^{T}-\tilde{e}_{5} R_{3} \tilde{e}_{5}^{T}\right)\right\} \xi(t), \\
E\left\{\dot{V}_{4}(t)\right\} & =\dot{e}^{T}(t)\left(\bar{\alpha} h R_{4}+(1-\bar{\alpha}) h R_{5}\right) \dot{e}(t)-\int_{t-\bar{\alpha} h}^{t} \dot{e}^{T}(s) R_{4} \dot{e}(s) d s-\int_{t-h}^{t-\bar{\alpha} h} \dot{e}^{T}(s) R_{5} \dot{e}(s) d s .
\end{aligned}
$$

By Lemma 2.3, for any positive scalars $\mu_{i}(i=1,2,3,4)$ satisfying $\mu_{1}+\mu_{2}=1$ and $\mu_{3}+\mu_{4}=1$, we have

$$
\begin{aligned}
& -\int_{t-\bar{\alpha} h}^{t} \dot{e}^{T}(s) R_{4} \dot{e}(s) d s \\
= & -\mu_{1} \int_{t-\bar{\alpha} h}^{t} \dot{e}^{T}(s) R_{4} \dot{e}(s) d s-\mu_{2} \int_{t-\bar{\alpha} h}^{t} \dot{e}^{T}(s) R_{4} \dot{e}(s) d s \\
\leq & -\left[\begin{array}{c}
e(t) \\
e(t-\bar{\alpha} h) \\
\int_{t-\bar{\alpha} h}^{t} e(s) d s
\end{array}\right]^{T}\left(\Omega_{1}\left(\mu_{1}, \mu_{2}, \bar{\alpha} h\right) \otimes R_{4}\right)\left[\begin{array}{c}
e(t) \\
e(t-\bar{\alpha} h) \\
\int_{t-\bar{\alpha} h}^{t} e(s) d s
\end{array}\right], \\
& -\int_{t-h}^{t-\bar{\alpha} h} \dot{e}^{T}(s) R_{5} \dot{e}(s) d s \\
= & -\mu_{3} \int_{t-h}^{t-\bar{\alpha} h} \dot{e}^{T}(s) R_{5} \dot{e}(s) d s-\mu_{4} \int_{t-h}^{t-\bar{\alpha} h} \dot{e}^{T}(s) R_{5} \dot{e}(s) d s \\
\leq & -\left[\begin{array}{c}
e(t-\bar{\alpha} h) \\
e(t-h) \\
\int_{t-h}^{t-\bar{\alpha} h} e(s) d s
\end{array}\right]^{T}\left(\Omega_{1}\left(\mu_{3}, \mu_{4},(1-\bar{\alpha}) h\right) \otimes R_{5}\right)\left[\begin{array}{c}
e(t-\bar{\alpha} h) \\
e(t-h) \\
\int_{t-h}^{t-\bar{\alpha} h} e(s) d s
\end{array}\right] .
\end{aligned}
$$

Considering (23)-(25), we obtain

$$
\begin{aligned}
E\left\{\dot{V}_{4}(t)\right\} & \leq \xi^{T}(t)\left\{\frac { t _ { l ^ { ( j + 1 ) } } - t } { \tilde { T } _ { k + 1 + n _ { j , j + 1 } } } \left(\tilde{e}_{2}\left(\bar{\alpha} h R_{4}+(1-\bar{\alpha}) h R_{5}\right) \tilde{e}_{2}^{T}-\left[\tilde{e}_{1}, \tilde{e}_{4}, \tilde{e}_{9}\right]\left(\Omega_{1}\left(\mu_{1}, \mu_{2}, \bar{\alpha} h\right) \otimes R_{4}\right)\left[\tilde{e}_{1}, \tilde{e}_{4}, \tilde{e}_{9}\right]^{T}\right.\right. \\
& \left.-\left[\tilde{e}_{4}, \tilde{e}_{5}, \tilde{e}_{10}\right]\left(\Omega_{1}\left(\mu_{3}, \mu_{4},(1-\bar{\alpha}) h\right) \otimes R_{5}\right)\left[\tilde{e}_{4}, \tilde{e}_{5}, \tilde{e}_{10}\right]^{T}\right) \\
& +\frac{t-t_{l^{(j)}}}{\tilde{T}_{k+1+n_{j, j+1}}}\left(\tilde{e}_{2}\left(\bar{\alpha} h R_{4}+(1-\bar{\alpha}) h R_{5}\right) \tilde{e}_{2}^{T}-\left[\tilde{e}_{1}, \tilde{e}_{4}, \tilde{e}_{9}\right]\left(\Omega_{1}\left(\mu_{1}, \mu_{2}, \bar{\alpha} h\right) \otimes R_{4}\right)\left[\tilde{e}_{1}, \tilde{e}_{4}, \tilde{e}_{9}\right]^{T}\right. \\
& \left.\left.-\left[\tilde{e}_{4}, \tilde{e}_{5}, \tilde{e}_{10}\right]\left(\Omega_{1}\left(\mu_{3}, \mu_{4},(1-\bar{\alpha}) h\right) \otimes R_{5}\right)\left[\tilde{e}_{4}, \tilde{e}_{5}, \tilde{e}_{10}\right]^{T}\right)\right\} \xi(t) .
\end{aligned}
$$




$$
E\left\{\dot{V}_{5}(t)\right\}=\dot{e}^{T}(t)\left(\frac{\bar{\alpha}^{4} h^{2}}{4} R_{6}+\frac{h^{2}}{4} R_{7}\right) \dot{e}(t)-\frac{\bar{\alpha}^{2}}{2} \int_{t-\bar{\alpha} h}^{t} \int_{\theta}^{t} \dot{e}^{T}(s) R_{6} \dot{e}(s) d s d \theta-\frac{1}{2} \int_{t-h}^{t} \int_{\theta}^{t} \dot{e}^{T}(s) R_{7} \dot{e}(s) d s d \theta .
$$

By Lemma 2.2, we have

$$
\begin{aligned}
& -\frac{\bar{\alpha}^{2}}{2} \int_{t-\bar{\alpha} h}^{t} \int_{\theta}^{t} \dot{e}^{T}(s) R_{6} \dot{e}(s) d s d \theta \\
& \leq-\left[\begin{array}{c}
e(t) \\
\int_{t-\bar{\alpha} h}^{t} e(s) d s \\
\int_{t-\bar{\alpha} h}^{t} \int_{\theta}^{t} e(s) d s d \theta
\end{array}\right]^{T}\left(\Omega_{2}(\bar{\alpha} h) \otimes R_{6}\right)\left[\begin{array}{c}
e(t) \\
\int_{t-\bar{\alpha} h}^{t} e(s) d s \\
\int_{t-\bar{\alpha} h}^{t} \int_{\theta}^{t} e(s) d s d \theta
\end{array}\right], \\
& -\frac{1}{2} \int_{t-h}^{t} \int_{\theta}^{t} \dot{e}^{T}(s) R_{7} \dot{e}(s) d s d \theta \\
& \leq-\left[\begin{array}{c}
e(t) \\
\int_{t-\bar{\alpha} h}^{t} e(s) d s+\int_{t-h}^{t-\bar{\alpha} h} e(s) d s \\
\int_{t-h}^{t} \int_{\theta}^{t} e(s) d s d \theta
\end{array}\right]^{T}\left(\Omega_{2}(h) \otimes R_{7}\right)\left[\begin{array}{c}
e(t) \\
\int_{t-\bar{\alpha} h}^{t} e(s) d s+\int_{t-h}^{t-\bar{\alpha} h} e(s) d s \\
\int_{t-h}^{t} \int_{\theta}^{t} e(s) d s d \theta
\end{array}\right] .
\end{aligned}
$$

Considering (27)-(29), we obtain

$$
\begin{aligned}
& E\left\{\dot{V}_{5}(t)\right\} \leq \xi^{T}(t)\left\{\frac { t _ { l ^ { ( j + 1 ) } } - t } { \tilde { T } _ { k + 1 + n _ { j , j + 1 } } } \left(\tilde{e}_{2}\left(\frac{\bar{\alpha}^{4} h^{2}}{4} R_{6}+\frac{h^{2}}{4} R_{7}\right) \tilde{e}_{2}^{T}-\left[\tilde{e}_{1}, \tilde{e}_{9}, \tilde{e}_{11}\right]\left(\Omega_{2}(\bar{\alpha} h) \otimes R_{6}\right)\left[\tilde{e}_{1}, \tilde{e}_{9}, \tilde{e}_{11}\right]^{T}\right.\right. \\
& \left.-\left[\tilde{e}_{1}, \tilde{e}_{9}+\tilde{e}_{10}, \tilde{e}_{12}\right]\left(\Omega_{2}(h) \otimes R_{7}\right)\left[\tilde{e}_{1}, \tilde{e}_{9}+\tilde{e}_{10}, \tilde{e}_{12}\right]^{T}\right) \\
& +\frac{t-t_{l(j)}}{\tilde{T}_{k+1+n_{j, j+1}}}\left(\tilde{e}_{2}\left(\frac{\bar{\alpha}^{4} h^{2}}{4} R_{6}+\frac{h^{2}}{4} R_{7}\right) \tilde{e}_{2}^{T}-\left[\tilde{e}_{1}, \tilde{e}_{9}, \tilde{e}_{11}\right]\left(\Omega_{2}(\bar{\alpha} h) \otimes R_{6}\right)\left[\tilde{e}_{1}, \tilde{e}_{9}, \tilde{e}_{11}\right]^{T}\right. \\
& \left.\left.-\left[\tilde{e}_{1}, \tilde{e}_{9}+\tilde{e}_{10}, \tilde{e}_{12}\right]\left(\Omega_{2}(h) \otimes R_{7}\right)\left[\tilde{e}_{1}, \tilde{e}_{9}+\tilde{e}_{10}, \tilde{e}_{12}\right]^{T}\right)\right\} \xi(t) . \\
& E\left\{\dot{V}_{6}(t)\right\}=2 \sum_{s=1}^{m_{f}}\left(\omega_{s}-\iota_{s}\right) g_{s}\left(d s^{T} e(t)\right) d s^{T} \dot{e}(t)+2 \sum_{s=1}^{m_{f}}\left(\iota_{s} k_{s}^{+}-\omega_{s} k_{s}^{-}\right) d s^{T} e(t) d s^{T} \dot{e}(t) \\
& =2 \dot{e}^{T}(t) D^{T}\left(L_{1}-L_{2}\right) g(t)+2 e^{T}(t) D^{T}\left(\bar{K}^{+} L_{2}-\bar{K}^{-} L_{1}\right) D \dot{e}(t) \\
& =\xi^{T}(t)\left\{2 \frac{t_{l^{(j+1)}}-t}{\tilde{T}_{k+1+n_{j, j+1}}}\left(\tilde{e}_{2} D^{T}\left(L_{1}-L_{2}\right) \tilde{e}_{6}^{T}+\tilde{e}_{1} D^{T}\left(\bar{K}^{+} L_{2}-\bar{K}^{-} L_{1}\right) D \tilde{e}_{2}^{T}\right)\right. \\
& \left.+2 \frac{t-t_{l^{(j)}}}{\tilde{T}_{k+1+n_{j, j+1}}}\left(\tilde{e}_{2} D^{T}\left(L_{1}-L_{2}\right) \tilde{e}_{6}^{T}+\tilde{e}_{1} D^{T}\left(\bar{K}^{+} L_{2}-\bar{K}^{-} L_{1}\right) D \tilde{e}_{2}^{T}\right)\right\} \xi(t), \\
& E\left\{\dot{V}_{7}(t)\right\}=\sum_{i=1}^{m} \theta_{i} e^{T}\left(t-\rho_{i-1}\right) Q_{i} e\left(t-\rho_{i-1}\right)-\sum_{i=1}^{m} \theta_{i} e^{T}\left(t-\lambda_{i} \rho_{i}\right) Q_{i} e\left(t-\lambda_{i} \rho_{i}\right) \\
& +\sum_{i=1}^{m} \theta_{i} \bar{\rho}_{i}^{2} \dot{e}^{T}(t) Z_{i} \dot{e}(t)-\sum_{i=1}^{m} \theta_{i} \bar{\rho}_{i} \int_{t-\lambda_{i} \rho_{i}}^{t-\rho_{i-1}} \dot{e}^{T}(s) Z_{i} \dot{e}(s) d s \\
& =\theta_{1} e^{T}(t) Q_{1} e(t)+\sum_{i=1}^{m-1} e^{T}\left(t-\rho_{i}\right)\left(\theta_{i+1} Q_{i+1}-\theta_{i} Q_{i}\right) e\left(t-\rho_{i}\right)-\theta_{m} e^{T}\left(t-\lambda_{m} \rho_{m}\right) Q_{m} e\left(t-\lambda_{m} \rho_{m}\right) \\
& +\sum_{i=1}^{m} \theta_{i} \bar{\rho}_{i}^{2} \dot{e}^{T}(t) Z_{i} \dot{e}(t)-\sum_{i=1}^{m} \theta_{i} \bar{\rho}_{i} \int_{t-\lambda_{i} \rho_{i}}^{t-\rho_{i-1}} \dot{e}^{T}(s) Z_{i} \dot{e}(s) d s .
\end{aligned}
$$

By adopting the definition of reciprocally convex combination and the lower bounds theorem in [37], which lead to

$$
-\sum_{i=1}^{m} \theta_{i} \bar{\rho}_{i} \int_{t-\lambda_{i} \rho_{i}}^{t-\rho_{i-1}} \dot{e}^{T}(s) S_{1 i} \dot{e}(s) d s \leq \sum_{i=1}^{m} \theta_{i} \eta_{1 i}^{T} \mathcal{S}_{1 i} \eta_{1 i} .
$$


By applying Lemma 2.1, we can get

$$
-\sum_{i=1}^{m} \theta_{i} \bar{\rho}_{i} \int_{t-\lambda_{i} \rho_{i}}^{t-\rho_{i-1}} \dot{e}^{T}(s) S_{2 i} \dot{e}(s) d s \leq \sum_{i=1}^{m} \theta_{i} \eta_{2 i}^{T} \mathcal{S}_{2 i} \eta_{2 i} .
$$

Considering (32)-(34), we obtain

$$
\begin{aligned}
E\left\{\dot{V}_{7}(t)\right\} \leq & \xi^{T}(t)\left\{\frac { t _ { l ( j + 1 ) } - t } { \tilde { T } _ { k + 1 + n _ { j , j + 1 } } } \left(\theta_{1} \tilde{e}_{1} Q_{1} \tilde{e}_{1}^{T}+\sum_{i=1}^{m-1} \tilde{e}_{11+3 i}\left(\theta_{i+1} Q_{i+1}-\theta_{i} Q_{i}\right) \tilde{e}_{11+3 i}^{T}-\theta_{m} \tilde{e}_{11+3 m} Q_{m} \tilde{e}_{11+3 m}^{T}\right.\right. \\
& +\sum_{i=1}^{m} \theta_{i} \bar{\rho}_{i}^{2} \tilde{e}_{2} Z_{i} \tilde{e}_{2}^{T}+\theta_{1}\left[\tilde{e}_{1}, \tilde{e}_{13}, \tilde{e}_{14}\right] \mathcal{S}_{11}\left[\tilde{e}_{1}, \tilde{e}_{13}, \tilde{e}_{14}\right]^{T} \\
& +\sum_{i=2}^{m} \theta_{i}\left[\tilde{e}_{8+3 i}, \tilde{e}_{10+3 i}, \tilde{e}_{11+3 i}\right] \mathcal{S}_{1 i}\left[\tilde{e}_{8+3 i}, \tilde{e}_{10+3 i}, \tilde{e}_{11+3 i}\right]^{T} \\
& +\theta_{1}\left[\tilde{e}_{1}, \tilde{e}_{14}, \tilde{e}_{15}\right] \mathcal{S}_{21}\left[\tilde{e}_{1}, \tilde{e}_{14}, \tilde{e}_{15}\right]^{T} \\
& \left.+\sum_{i=2}^{m} \theta_{i}\left[\tilde{e}_{8+3 i}, \tilde{e}_{11+3 i}, \tilde{e}_{12+3 i}\right] \mathcal{S}_{2 i}\left[\tilde{e}_{8+3 i}, \tilde{e}_{11+3 i}, \tilde{e}_{12+3 i}\right]^{T}\right) \\
& +\frac{t-t_{l(j)}}{\tilde{T}_{k+1+n_{j, j+1}}}\left(\theta_{1} \tilde{e}_{1} Q_{1} \tilde{e}_{1}^{T}+\sum_{i=1}^{m-1} \tilde{e}_{11+3 i}\left(\theta_{i+1} Q_{i+1}-\theta_{i} Q_{i}\right) \tilde{e}_{11+3 i}^{T}-\theta_{m} \tilde{e}_{11+3 m} Q_{m} \tilde{e}_{11+3 m}^{T}\right. \\
& +\sum_{i=1}^{m} \theta_{i} \bar{\rho}_{i}^{2} \tilde{e}_{2} Z_{i} \tilde{e}_{2}^{T}+\theta_{1}\left[\tilde{e}_{1}, \tilde{e}_{13}, \tilde{e}_{14}\right] \mathcal{S}_{11}\left[\tilde{e}_{1}, \tilde{e}_{13}, \tilde{e}_{14}\right]^{T} \\
& +\sum_{i=2}^{m} \theta_{i}\left[\tilde{e}_{8+3 i}, \tilde{e}_{10+3 i}, \tilde{e}_{11+3 i}\right] \mathcal{S}_{1 i}\left[\tilde{e}_{8+3 i}, \tilde{e}_{10+3 i}, \tilde{e}_{11+3 i}\right]^{T} \\
& +\theta_{1}\left[\tilde{e}_{1}, \tilde{e}_{14}, \tilde{e}_{15}\right] \mathcal{S}_{21}\left[\tilde{e}_{1}, \tilde{e}_{14}, \tilde{e}_{15}\right]^{T} \\
& \left.\left.+\sum_{i=2}^{m} \theta_{i}\left[\tilde{e}_{8+3 i}, \tilde{e}_{11+3 i}, \tilde{e}_{12+3 i}\right] \mathcal{S}_{2 i}\left[\tilde{e}_{8+3 i}, \tilde{e}_{11+3 i}, \tilde{e}_{12+3 i}\right]^{T}\right)\right\} \xi(t) .
\end{aligned}
$$

In addition, notice that $E\left\{\alpha_{l}(t)\right\}=\theta_{l}(l=1,2, \ldots, m)$, then, for any scalars $y_{1}, y_{2}$ and $y_{3}$, and arbitrary matrix $N$ with appropriate dimension, the following equality holds:

$$
\begin{aligned}
0= & E\left\{2 [ \dot { e } ^ { T } ( t ) y _ { 1 } + e ^ { T } ( t ) y _ { 2 } + e ^ { T } ( t _ { l ^ { ( j ) } } ) y _ { 3 } ] N \left[-\dot{e}(t)+A_{q} e(t)+B_{q} e(t-h)\right.\right. \\
& \left.\left.+W_{q} g(t)-\sum_{l=1}^{m} \alpha_{l}(t) K C_{q} e\left(t-\bar{\rho}_{l}(t)\right)\right]\right\} \\
= & \xi^{T}(t)\left\{2 \frac{t_{l^{(j+1)}}-t}{\tilde{T}_{k+1+n_{j, j+1}}}\left[y_{1} \tilde{e}_{2}+y_{2} \tilde{e}_{1}+y_{3} \tilde{e}_{3}\right] N\left[-\tilde{e}_{2}^{T}+A_{q} \tilde{e}_{1}^{T}+B_{q} \tilde{e}_{5}^{T}+W_{q} \tilde{e}_{6}^{T}-\sum_{l=1}^{m} \theta_{l} K C_{q} \tilde{e}_{10+3 l}^{T}\right]\right. \\
& \left.+\frac{t-t_{l^{(j)}}}{\tilde{T}_{k+1+n_{j, j+1}}}\left[y_{1} \tilde{e}_{2}+y_{2} \tilde{e}_{1}+y_{3} \tilde{e}_{3}\right] N\left[-\tilde{e}_{2}^{T}+A_{q} \tilde{e}_{1}^{T}+B_{q} \tilde{e}_{5}^{T}+W_{q} \tilde{e}_{6}^{T}-\sum_{l=1}^{m} \theta_{l} K C_{q} \tilde{e}_{10+3 l}^{T}\right]\right\} \xi(t), \\
= & \xi^{T}(t)\left\{2 \frac{t_{l^{(j+1)}}-t}{\tilde{T}_{k+1+n_{j, j+1}}}\left[y_{1} \tilde{e}_{2}+y_{2} \tilde{e}_{1}+y_{3} \tilde{e}_{3}\right]\left[-N \tilde{e}_{2}^{T}+N A_{q} \tilde{e}_{1}^{T}+N B_{q} \tilde{e}_{5}^{T}+N W_{q} \tilde{e}_{6}^{T}-\sum_{l=1}^{m} \theta_{l} U C_{q} \tilde{e}_{10+3 l}^{T}\right]\right. \\
& \left.+\frac{t-t_{l^{(j)}}}{\tilde{T}_{k+1+n_{j, j+1}}}\left[y_{1} \tilde{e}_{2}+y_{2} \tilde{e}_{1}+y_{3} \tilde{e}_{3}\right]\left[-N \tilde{e}_{2}^{T}+N A_{q} \tilde{e}_{1}^{T}+N B_{q} \tilde{e}_{5}^{T}+N W_{q} \tilde{e}_{6}^{T}-\sum_{l=1}^{m} \theta_{l} U C_{q} \tilde{e}_{10+3 l}^{T}\right]\right\} \xi(t) .
\end{aligned}
$$

According to (7), for any positive diagonal matrices $V_{1 i}=\operatorname{diag}\left\{v_{11 i}, v_{12 i}, \ldots, v_{1 m_{f} i}\right\}, V_{2 i}=\operatorname{diag}\left\{v_{21 i}, v_{22 i}, \ldots, v_{2 m_{f} i}\right\}$, and $V_{3 i}=\operatorname{diag}\left\{v_{31 i}, v_{32 i}, \ldots, v_{3 m_{f} i}\right\}(\mathrm{i}=1,2)$, we can get the following inequalities

$$
-2 \sum_{s=1}^{m_{f}}\left[g_{s}(t)-k_{s}^{+} d_{s}^{T} e(t)\right] v_{1 s i}\left[g_{s}(t)-k_{s}^{-} d_{s}^{T} e(t)\right] \geq 0
$$




$$
\begin{aligned}
& -2 \sum_{s=1}^{m_{f}}\left[g_{s}(t-\bar{\alpha} h)-k_{s}^{+} d_{s}^{T} e(t-\bar{\alpha} h)\right] v_{2 s i}\left[g_{s}(t-\bar{\alpha} h)-k_{s}^{-} d_{s}^{T} e(t-\bar{\alpha} h)\right] \geq 0, \\
& -2 \sum_{s=1}^{m_{f}}\left[g_{s}(t-h)-k_{s}^{+} d_{s}^{T} e(t-h)\right] v_{3 s i}\left[g_{s}(t-h)-k_{s}^{-} d_{s}^{T} e(t-h)\right] \geq 0,
\end{aligned}
$$

From (37)-(39), we have

$$
\begin{aligned}
\Phi_{1}(i, t)= & -2 g^{T}(t) V_{1 i} g(t)+2 e^{T}(t) D^{T}\left[\bar{K}^{+}+\bar{K}^{-}\right] V_{1 i} g(t)-2 e^{T}(t) D^{T} \bar{K}^{+} V_{1 i} \bar{K}^{-} D e(t) \geq 0, \\
\Phi_{2}(i, t)= & -2 g^{T}(t-\bar{\alpha} h) V_{2 i} g(t-\bar{\alpha} h)+2 e^{T}(t-\bar{\alpha} h) D^{T}\left[\bar{K}^{+}+\bar{K}^{-}\right] V_{2 i} g(t-\bar{\alpha} h) \\
& -2 e^{T}(t-\bar{\alpha} h) D^{T} \bar{K}^{+} V_{2 i} \bar{K}^{-} D e(t-\bar{\alpha} h) \geq 0, \\
\Phi_{3}(i, t)= & -2 g^{T}(t-h) V_{3 i} g(t-h)+2 e^{T}(t-h) D^{T}\left[\bar{K}^{+}+\bar{K}^{-}\right] V_{3 i} g(t-h) \\
& -2 e^{T}(t-h) D^{T} \bar{K}^{+} V_{3 i} \bar{K}^{-} D e(t-h) \geq 0 .
\end{aligned}
$$

Then,

$$
\begin{aligned}
& \frac{t_{l(j+1)}-t}{\tilde{T}_{k+1+n_{j, j+1}}} \Phi_{1}(1, t)+\frac{t-t_{l(j)}}{\tilde{T}_{k+1+n_{j, j+1}}} \Phi_{1}(2, t) \\
= & \xi^{T}(t)\left\{\frac{t_{l(j+1)}-t}{\tilde{T}_{k+1+n_{j, j+1}}}\left(-2 \tilde{e}_{6} V_{11} \tilde{e}_{6}^{T}+2 \tilde{e}_{1} D^{T}\left[\bar{K}^{+}+\bar{K}^{-}\right] V_{11} \tilde{e}_{6}^{T}-2 \tilde{e}_{1} D^{T} \bar{K}^{+} V_{11} \bar{K}^{-} D \tilde{e}_{1}^{T}\right)+\right. \\
& \left.\frac{t-t_{l^{(j)}}}{\tilde{T}_{k+1+n_{j, j+1}}}\left(-2 \tilde{e}_{6} V_{12} \tilde{e}_{6}^{T}+2 \tilde{e}_{1} D^{T}\left[\bar{K}^{+}+\bar{K}^{-}\right] V_{12} \tilde{e}_{6}^{T}-2 \tilde{e}_{1} D^{T} \bar{K}^{+} V_{12} \bar{K}^{-} D \tilde{e}_{1}^{T}\right)\right\} \xi(t) \geq 0, \\
& \frac{t_{l(j+1)}-t}{\tilde{T}_{k+1+n_{j, j+1}}} \Phi_{2}(1, t)+\frac{t-t_{l(j)}}{\tilde{T}_{k+1+n_{j, j+1}}} \Phi_{2}(2, t) \\
= & \xi^{T}(t)\left\{\frac{t_{l^{(j+1)}}-t}{\tilde{T}_{k+1+n_{j, j+1}}}\left(-2 \tilde{e}_{7} V_{21} \tilde{e}_{7}^{T}+2 \tilde{e}_{4} D^{T}\left[\bar{K}^{+}+\bar{K}^{-}\right] V_{21} \tilde{e}_{7}^{T}-2 \tilde{e}_{4} D^{T} \bar{K}^{+} V_{21} \bar{K}^{-} D \tilde{e}_{4}^{T}\right)+\right. \\
& \left.\frac{t-t_{l^{(j)}}}{\tilde{T}_{k+1+n_{j, j+1}}}\left(-2 \tilde{e}_{7} V_{22} \tilde{e}_{7}^{T}+2 \tilde{e}_{4} D^{T}\left[\bar{K}^{+}+\bar{K}^{-}\right] V_{22} \tilde{e}_{7}^{T}-2 \tilde{e}_{4} D^{T} \bar{K}^{+} V_{22} \bar{K}^{-} D \tilde{e}_{4}^{T}\right)\right\} \xi(t) \geq 0, \\
& \frac{t_{l(j+1)}-t}{\tilde{T}_{k+1+n_{j, j+1}}} \Phi_{3}(1, t)+\frac{t-t_{l^{(j)}}}{\tilde{T}_{k+1+n_{j, j+1}}} \Phi_{3}(2, t) \\
= & \xi^{T}(t)\left\{\frac{t_{l^{(j+1)}}-t}{\tilde{T}_{k+1+n_{j, j+1}}}\left(-2 \tilde{e}_{8} V_{31} \tilde{e}_{8}^{T}+2 \tilde{e}_{5} D^{T}\left[\bar{K}^{+}+\bar{K}^{-}\right] V_{31} \tilde{e}_{8}^{T}-2 \tilde{e}_{5} D^{T} \bar{K}^{+} V_{31} \bar{K}^{-} D \tilde{e}_{5}^{T}\right)+\right. \\
& \left.\frac{t-t_{l(j)}}{\tilde{T}_{k+1+n_{j, j+1}}}\left(-2 \tilde{e}_{8} V_{32} \tilde{e}_{8}^{T}+2 \tilde{e}_{5} D^{T}\left[\bar{K}^{+}+\bar{K}^{-}\right] V_{32} \tilde{e}_{8}^{T}-2 \tilde{e}_{5} D^{T} \bar{K}^{+} V_{32} \bar{K}^{-} D \tilde{e}_{5}^{T}\right)\right\} \xi(t) \geq 0 .
\end{aligned}
$$

Considering (18), (21), (22), (26), (30), (31), (35), (36) and (40)-(42), we obtain

$$
E\{\dot{V}(t)\} \leq \xi^{T}(t)\left\{\frac{t_{l^{(j+1)}}-t}{\tilde{T}_{k+1+n_{j, j+1}}} \Gamma_{1}\left(\tilde{T}_{k+1+n_{j, j+1}}\right)+\frac{t-t_{l^{(j)}}}{\tilde{T}_{k+1+n_{j, j+1}}} \Gamma_{2}\left(\tilde{T}_{k+1+n_{j, j+1}}\right)\right\} \xi(t) .
$$

It noted that

$$
\Gamma_{1}\left(\tilde{T}_{k+1+n_{j, j+1}}\right)=\frac{\tilde{T}_{k+1+n_{j, j+1}}}{(\bar{N}+1) \rho_{m}} \Gamma_{1}\left((\bar{N}+1) \rho_{m}\right)+\frac{(\bar{N}+1) \rho_{m}-\tilde{T}_{k+1+n_{j, j+1}}}{(\bar{N}+1) \rho_{m}} \Gamma_{1}(0),
$$

and

$$
\Gamma_{2}\left(\tilde{T}_{k+1+n_{j, j+1}}\right)=\frac{\tilde{T}_{k+1+n_{j, j+1}}}{(\bar{N}+1) \rho_{m}} \Gamma_{2}\left((\bar{N}+1) \rho_{m}\right)+\frac{(\bar{N}+1) \rho_{m}-\tilde{T}_{k+1+n_{j, j+1}}}{(\bar{N}+1) \rho_{m}} \Gamma_{2}(0) .
$$

From (15) and (16), we obtain that

$$
\Gamma_{1}\left(\tilde{T}_{k+1+n_{j, j+1}}\right)<0, \quad \Gamma_{2}(0)<0 .
$$


Based on Schur complement, we have from (16)

$$
\Gamma_{2}\left((\bar{N}+1) \rho_{m}\right)<0 .
$$

From (46) and (47), it can be seen that

$$
\Gamma_{2}\left(\tilde{T}_{k+1+n_{j, j+1}}\right)<0 .
$$

Then, we can obtain from (43), (46) and (48) that

$$
E\{\dot{V}(t)\} \leq-\epsilon\|e(t)\|^{2}, \quad t \in\left[t_{l^{(j)}}, t_{l^{(j+1)}}\right)
$$

for some $\epsilon>0$. Now, using Dynkin's formula, we have that

$$
E\left\{V\left(t_{l^{(j+1)}}\right)\right\}-E\left\{V\left(t_{l^{(j)}}\right)\right\}=E\left\{V\left(t_{l^{(j+1)}}^{-}\right)\right\}-E\left\{V\left(t_{l^{(j)}}\right)\right\} \leq-\epsilon E\left\{\int_{{ }_{l_{l}(j)}}^{t_{{ }^{(j+1)}}^{-}}\|e(s)\|^{2} d s\right\} .
$$

Thus, we can get from (50) that

$$
\sum_{j=0}^{\infty} E\left\{\int_{t_{l}(j)}^{t{ }^{(j+1)}}\|e(s)\|^{2} d s\right\} \leq \epsilon^{-1} E\left\{V\left(t_{l^{(0)}}\right)\right\} .
$$

Therefore, according to Definition 2.1, we have that the synchronization error system (12) is stochastically stable, that is, master system (1) and slave system (4) are stochastically synchronous. This completes the proof.

Remark 3.1. Due to the time-varying term $\pi_{q j}(\bar{h})$, it is difficult to solve inequalities (15) and (16), because they contain infinite number of inequalities, which limits the use of Theorem 3.1 in practice. To overcome this shortcoming, we present our next result which reduces infinite number of inequalities in Theorem 3.1 to finitely many ones, which will be simple to solve.

Theorem 3.2. For given scalars $h>0,0<\bar{\alpha}<1, \delta, 0 \leq \mu_{i_{1}} \leq 1\left(i_{1}=1,2,3,4\right), \mu_{1}+\mu_{2}=1, \mu_{3}+\mu_{4}=$ 1 , the master system (1) and the slave system (4) are stochastically synchronous if there exist matrices $P_{q}>0$, $R_{i_{2}}>0\left(i_{2}=1,2,3,4,5,6,7\right), Q_{i_{3}}>0, S_{1 i_{3}}>0, S_{2 i_{3}}>0\left(i_{3}=1,2, \ldots, m\right), L_{1}=\operatorname{diag}\left\{\omega_{1}, \omega_{2}, \ldots, \omega_{m}\right\}>0$, $L_{2}=\operatorname{diag}\left\{\iota_{1}, \iota_{2}, \ldots, \iota_{m}\right\}>0, V_{j l}=\operatorname{diag}\left\{v_{j 1 l}, v_{j 2 l}, \ldots, v_{j m_{f} l}\right\}>0(l=1,2 ; j=1,2,3)$, and any appropriately dimensioned matrices $\bar{W}_{i_{4}}\left(i_{4}=1,2, \ldots, m\right), H_{1}, H_{2}, N, U, M=\left[M_{1}, M_{2}, M_{3}\right], \bar{M}=[M, 0]$ and $T_{q j}(j \neq q, j \in \mathcal{L})$, such that (13), (14) and the following matrix inequalities hold for all $q \in \mathcal{L}$ :

$$
\begin{aligned}
\Gamma_{1}^{*}(\hat{T}) & =\left[\begin{array}{cc}
\Upsilon_{0}^{*}+\Upsilon_{01}+\Upsilon_{1}(\hat{T}) & \mathcal{P}_{q} \\
* & -\mathcal{T}_{q}
\end{array}\right]<0, \\
\hat{\Gamma}_{2}^{*}(\hat{T}) & =\left[\begin{array}{ccc}
\Upsilon_{0}^{*}+\Upsilon_{02} & \hat{T}^{\frac{1}{2}} \bar{M}^{T} & \mathcal{P}_{q} \\
* & -R_{1} & 0 \\
* & * & -\mathcal{T}_{q}
\end{array}\right]<0, \quad \hat{T}=0,(\bar{N}+1) \rho_{m} .
\end{aligned}
$$

where $\Upsilon_{0}^{*}, \Upsilon_{01}, \Upsilon_{02}$ and $\Upsilon_{1}(\hat{T})$ are defined in Appendix.

Moreover, the gain matrix is given by

$$
K=N^{-1} U .
$$


Proof. According to Theorem 3.1, we have

$$
\begin{aligned}
\Upsilon_{0} & =\check{\Upsilon}_{0}+\sum_{j=1}^{s} \pi_{q j}(h) \tilde{e}_{1} P_{j} \tilde{e}_{1}^{T} \\
& =\check{\Upsilon}_{0}+\tilde{e}_{1}\left\{\sum_{j=1}^{s} \pi_{q j} P_{j}+\sum_{j=1, j \neq q}^{s}\left[\frac{1}{2} \triangle \pi_{q j}\left(P_{j}-P_{q}\right)+\frac{1}{2} \triangle \pi_{q j}\left(P_{j}-P_{q}\right)\right]\right\} \tilde{e}_{1}^{T} .
\end{aligned}
$$

By Lemma 2.4, if there exist matrices $T_{q j}$ for all $\left|\triangle \pi_{q j}\right| \leq \tilde{\pi}_{q j}$, such that

$$
\tilde{\Upsilon}_{0}^{*}+\Upsilon_{01}+\Upsilon_{1}(\hat{T})<0
$$

and

$$
\left[\begin{array}{cc}
\tilde{\Upsilon}_{0}^{*}+\Upsilon_{02} & \hat{T}^{\frac{1}{2}} \bar{M}^{T} \\
* & -R_{1}
\end{array}\right]<0, \quad \hat{T}=0,(\bar{N}+1) \rho_{m} .
$$

In view of Schur complement, it can be seen that (54) and (55) is equivalent to the inequalities (52) and (53). Then, by this fact together with Theorem 3.1, we can conclude that the synchronization error system (12) is stochastically stable. This completes the proof.

Remark 3.2. Note that Theorem 3.2 gives the stochastically synchronous conditions for master system (1) and slave system (4). The results are expressed within the framework of LMIs, which can be easily verified by the MATLAB LMI Toolbox. Moreover, if (13), (14), (52) and (53) are feasible, the desired sampled-data feedback control gain $K$ can be readily obtained.

Remark 3.3. In switched systems, we often call each subsystem a mode, and say that control problems are to design a set of mode-dependent controllers or a mode-independent controller for the unforced system and find admissible switching signals such that the resulting system is stable and satisfies certain performance criteria. With an adaptation sense, mode-dependent controller design is less conservative. However, a very common assumption in the 'modedependent' context is that the controllers are switched synchronously with the switching of system modes, which is quite unpractical. Due to the fact that it inevitably takes some time to identify the system modes and apply the controller, there exists asynchronous switching in actual operation, i.e. the switching instants of the controllers exceed or lag behind those of the systems. Thus, it is necessary to consider asynchronous switching for efficient control design. So, it is noteworthy that the proposed method can be extended to the stochastic asynchronous problem of semi-Markovian jump chaotic Lur'e systems subject to multiple sampling periods. Meanwhile, the conservatism of the obtained results will be reduced. This will be the topic of our future study.

Remark 3.4. In this paper, LMIs approach is employed to derive the results, which can be easily solved by MATLAB LMI Tool box. In Theorem 3.2, the complexity of variables are $\left(1.5 m+s^{2}-s+11\right) n^{2}+(1.5 m+l+4) n+2 m+6 m_{f}$. However, when the size of inequalities and the number of decision variables gets bigger, the calculation time gets longer. In future work, we will both consider conservativeness and calculation complexity.

Remark 3.5. Nowadays, most of the practical control systems employ the digital signals to transmit the information in order to minimize the total cost. However, traditional researches paid more attention to the sampled-data control 
systems based on the assumption that the considered system is sampled with a constant sampling interval [7,8]. Due to the unpredictable network-induced phenomena, such as fluctuated network loads and sampling errors, the sampling period may often jitter inevitably. Packet dropout is one of the important issues, which results from transmission errors or congestion in the physical communication links or from buffer overflows, which can degrade system performance and possibly cause system instability. So we consider packet dropout and multiple sampling periods in this paper. Compared with the results in [7] where master-slave synchronization problem is investigated for chaotic delayed Lur'e systems, and the synchronization problem for chaotic Lur'e systems with time delays by using sampled-data control in [8], more practical factors such as packet dropout, multiple sampling periods and semi-Markov process are considered in the model of this paper. It is noted that, two $\left(t_{l^{(j)}}, t_{l^{(j+1)}}\right)$-dependent terms $\left(t_{l^{(j+1)}}-t\right) \varepsilon^{T}(t) \mathcal{H} \varepsilon(t)$ and $V_{2}(t)$ are introduced in (17), which make full use of the information available on the actual sampling pattern. So, we can obtain less conservative results than those in [7,8]. To the best of our knowledge, there is little information in the published literature about stochastic synchronization of semi-Markovian jump chaotic Lur'e systems with packet dropouts subject to multiple sampling periods. In this viewpoint, the system model investigated in the paper is comprehensive.

\section{Illustrative example}

In this section, we will demonstrate the advantages and effectiveness of the proposed methods in this paper via an example.

Example 4.1. Consider the following time-delay Chua's circuit with two modes (i.e. $r_{t} \in \mathcal{L}=\{1,2\}$ ) as the master system:

$$
\left\{\begin{array}{l}
\dot{x}_{1}(t)=a\left(r_{t}\right)\left(x_{2}(t)-m_{1}\left(r_{t}\right) x_{1}(t)+\tilde{f}\left(x_{1}(t)\right)\right)-c\left(r_{t}\right) x_{1}(t-h) \\
\dot{x}_{2}(t)=x_{1}(t)-x_{2}(t)+x_{3}(t)-c\left(r_{t}\right) x_{1}(t-h) \\
\dot{x}_{3}(t)=-b\left(r_{t}\right) x_{2}(t)+c\left(r_{t}\right)\left(2 x_{1}(t-h)-x_{3}(t-h)\right)
\end{array}\right.
$$

with the nonlinear characteristics

$$
\tilde{f}\left(x_{1}(t)\right)=0.5\left(m_{1}\left(r_{t}\right)-m_{0}\left(r_{t}\right)\right)\left(\left|x_{1}(t)+1\right|-\left|x_{1}(t)-1\right|\right) .
$$

It can be found that Chua's circuit can be represented in the time-delay Lur'e form with

$$
\begin{aligned}
& A\left(r_{t}\right)=\left[\begin{array}{ccc}
-a\left(r_{t}\right) m_{1}\left(r_{t}\right) & a\left(r_{t}\right) & 0 \\
1 & -1 & 1 \\
0 & -b\left(r_{t}\right) & 0
\end{array}\right], \quad B\left(r_{t}\right)=\left[\begin{array}{ccc}
-c\left(r_{t}\right) & 0 & 0 \\
-c\left(r_{t}\right) & 0 & 0 \\
2 c\left(r_{t}\right) & 0 & -c\left(r_{t}\right)
\end{array}\right], \\
& W\left(r_{t}\right)=\left[\begin{array}{c}
-a\left(r_{t}\right)\left(m_{0}\left(r_{t}\right)-m_{1}\left(r_{t}\right)\right) \\
0 \\
0
\end{array}\right], \quad D=\left[\begin{array}{l}
1 \\
0 \\
0
\end{array}\right]^{T}
\end{aligned}
$$

and $f\left(x_{1}(t)\right)=0.5\left(\left|x_{1}(t)+1\right|-\left|x_{1}(t)-1\right|\right)$ belonging to sector $[0,1]$. We choose parameters $m_{0}(1)=-\frac{1}{7}, m_{1}(1)=\frac{2}{7}$, $a(1)=7, b(1)=12.26, c(1)=0.1, m_{0}(2)=-\frac{2}{7}, m_{1}(2)=\frac{1}{7}, a(2)=9, b(2)=14.28, c(2)=0.1$ and the constant 


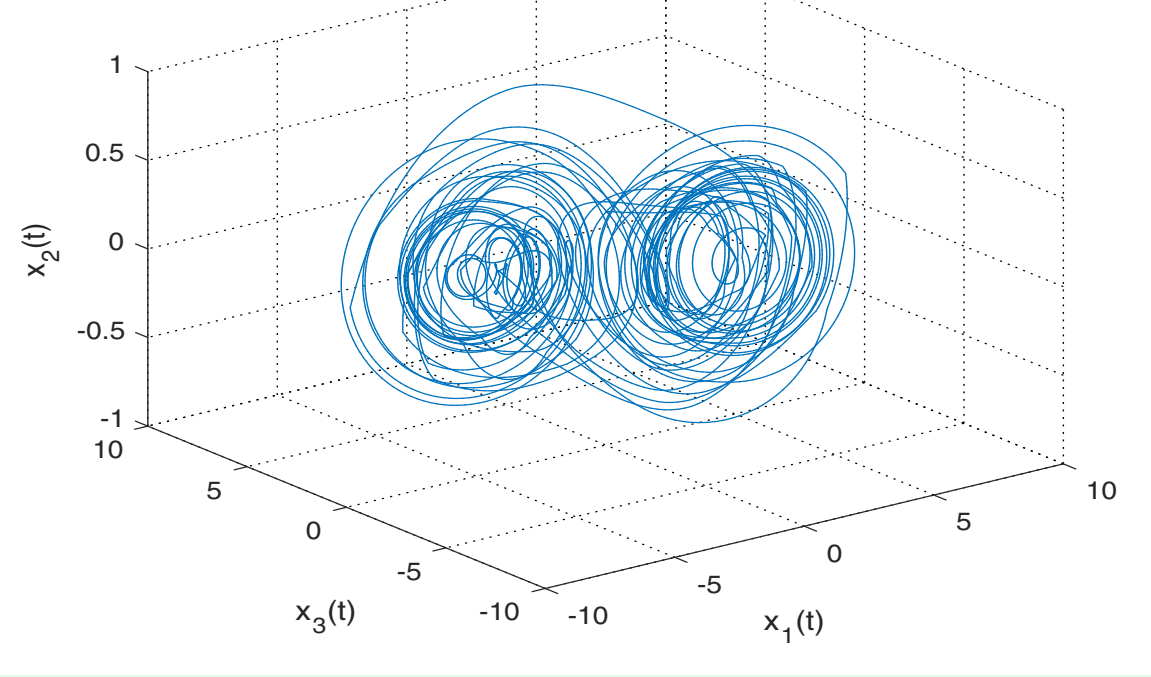

Figure 1: State trajectories of master system.

time delay $h=0.1$. In this example, the transition rates in the model are $\pi_{11}(\bar{h}) \in(-2.2,-1.8), \pi_{12}(\bar{h}) \in(1.8,2.2)$, $\pi_{21}(\bar{h}) \in(2.6,3.4), \pi_{22}(\bar{h}) \in(-3.4,-2.6)$, so we can always set $\pi_{11}=-2, \pi_{12}=2, \pi_{21}=3, \pi_{22}=-3$ and $\tilde{\pi}_{1 j}=0.2$, $\tilde{\pi}_{2 j}=0.4(j=1,2)$ for the stochastic stability analysis. And, we choose $C_{1}=C_{2}=\left[\begin{array}{lll}1 & 0 & 0\end{array}\right], \bar{N}=2, \rho_{1}=0.1$, $\rho_{2}=0.2, \beta_{1}=0.9, \beta_{2}=0.1, \bar{\theta}=0.1, \bar{\alpha}=0.7948, \mu_{1}=0.5085, \mu_{2}=0.4915, \mu_{3}=0.5108, \mu_{4}=0.4892, y_{1}=0.2886$, $y_{2}=-0.2428, y_{3}=0.6232$. In the case of $\bar{N}=2$, we can obtain $\theta_{1}=0.9002, \theta_{2}=0.0998$.

The initial conditions of the master and slave systems are chosen as $x(t)=[0.2,0.3,0.2]^{T}, y(t)=[-0.3,-0.1,0.4]^{T}$, $t \in[-0.2,0]$. Figure 1 and Figure 2 show the master system states $x(t)$ and the slave system states $y(t)$ with $u(t)=0$, respectively. Using the MATLAB LMI Toolbox to solve the LMIs (13), (14), (52) and (53), we can get the following gain matrix in (4):

$$
K=\left[\begin{array}{lll}
0.2831 & 0.2184 & -0.7137
\end{array}\right]^{T}
$$

That is, there exists a sampled-data controller such that the master and slave systems are stochastic synchronization. For the above gain matrix, the response curves of the master system (1), the slave system (4), the error system (12) and $r_{t}$ are given in Figure 3, Figure 4 , Figure 5 and Figure 6, respectively. Figure 5 shows that the synchronization error is tending to zero. Thus we can synchronize successfully the master and slave systems by the proposed sampleddata controller.

\section{Conclusion}

In this paper, the problem of stochastic synchronization has been discussed for semi-Markovian jump chaotic Lur'e systems. By input-delay approach and getting the utmost out of the usable information on the actual sampling pattern, some sufficient conditions in terms of LMIs are derived to ensure the stochastic stability of the error system. 


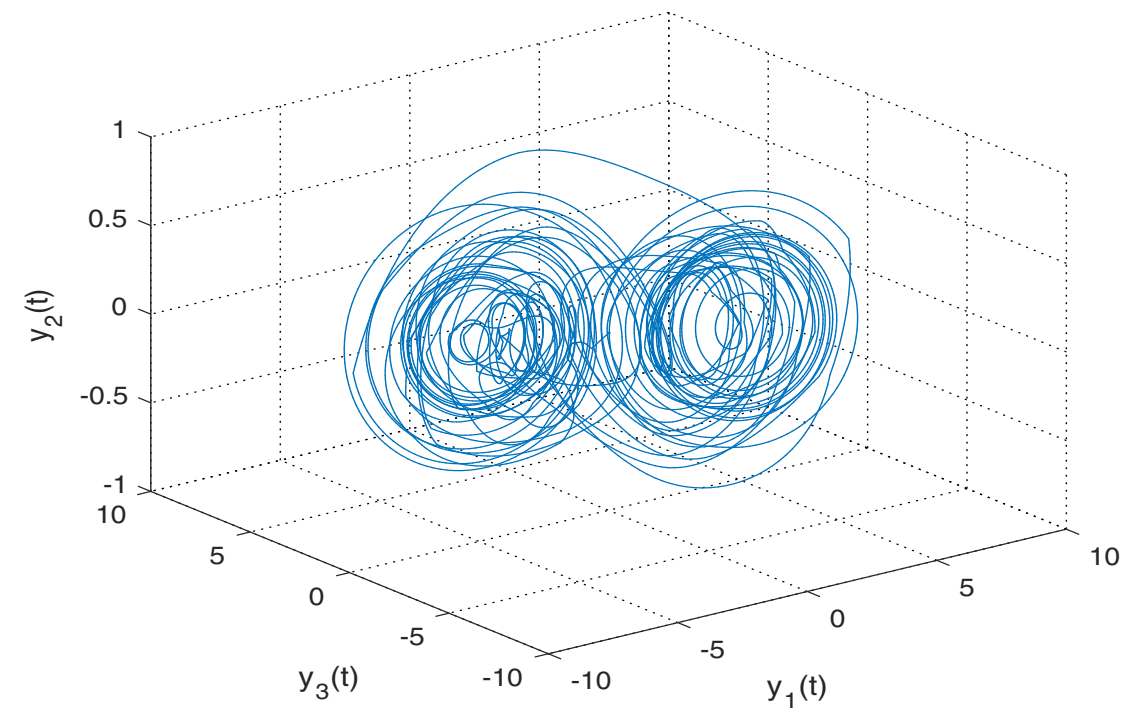

Figure 2: State trajectories of slave system without $u(t)$.

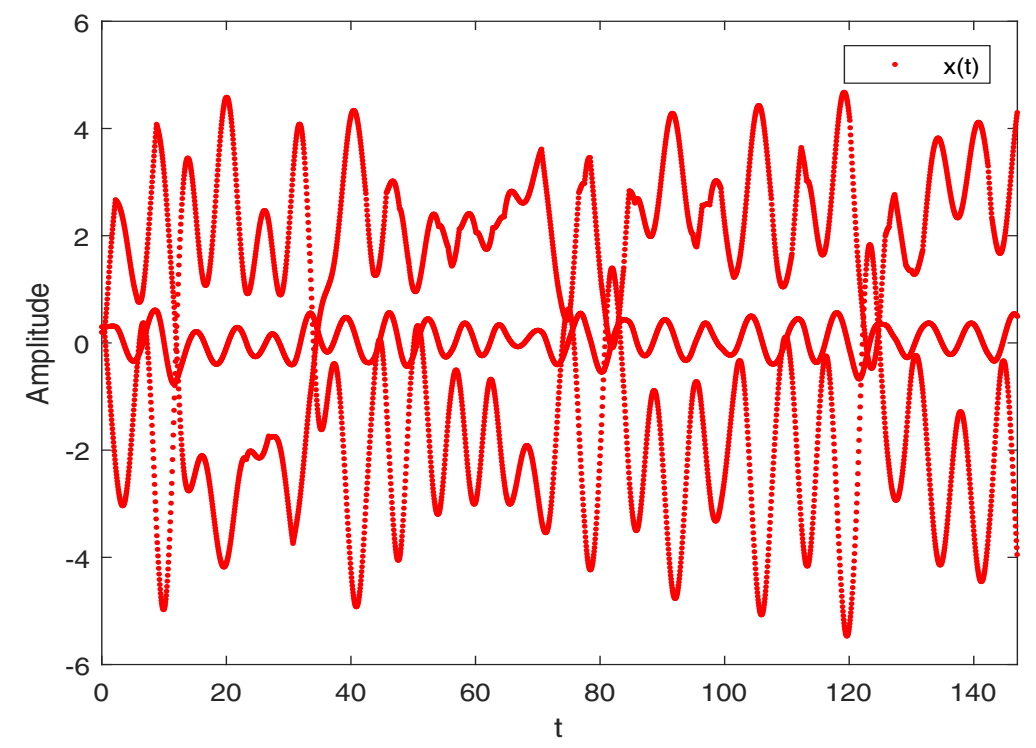

Figure 3: State trajectories of master system. 


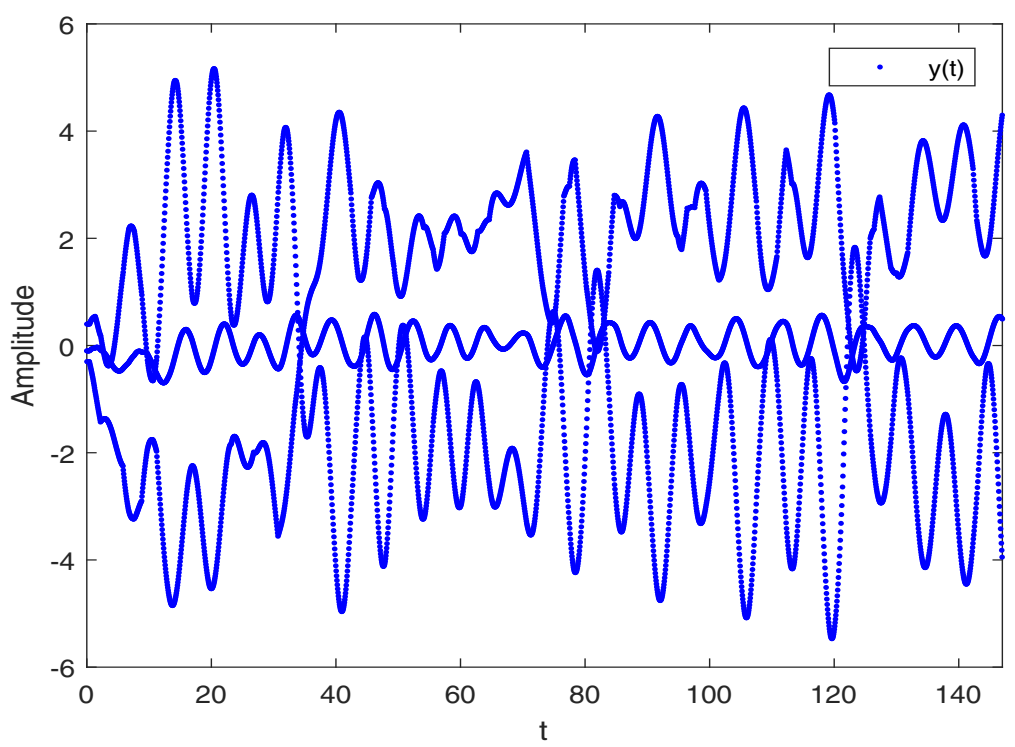

Figure 4: State trajectories of slave system.

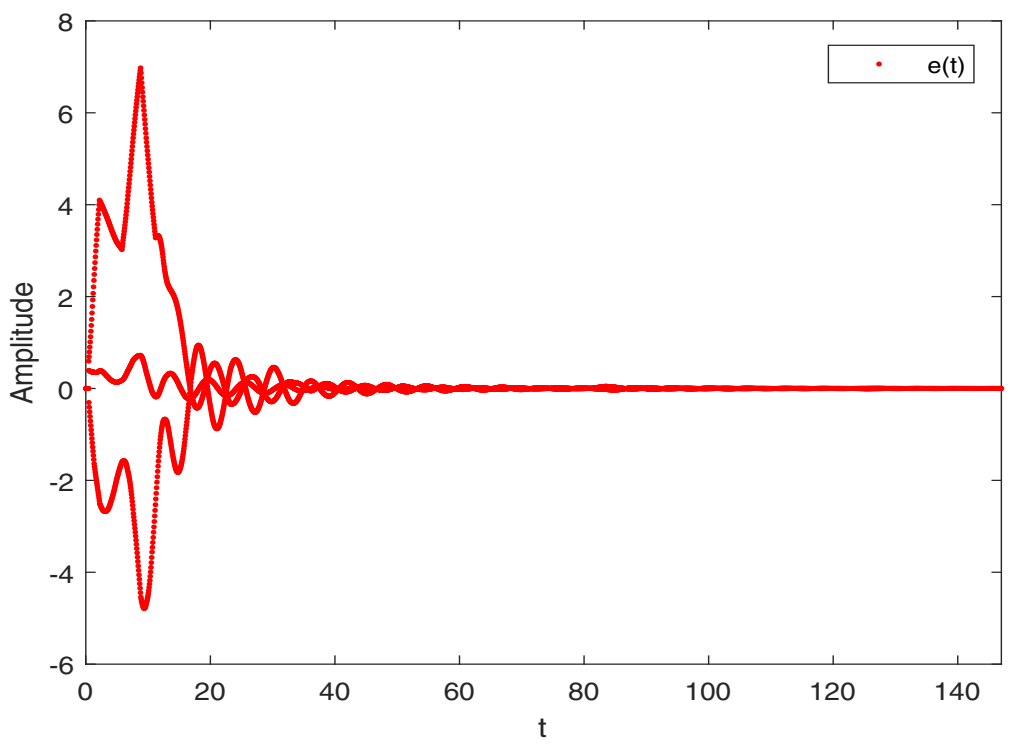

Figure 5: State trajectories of error system.

20 


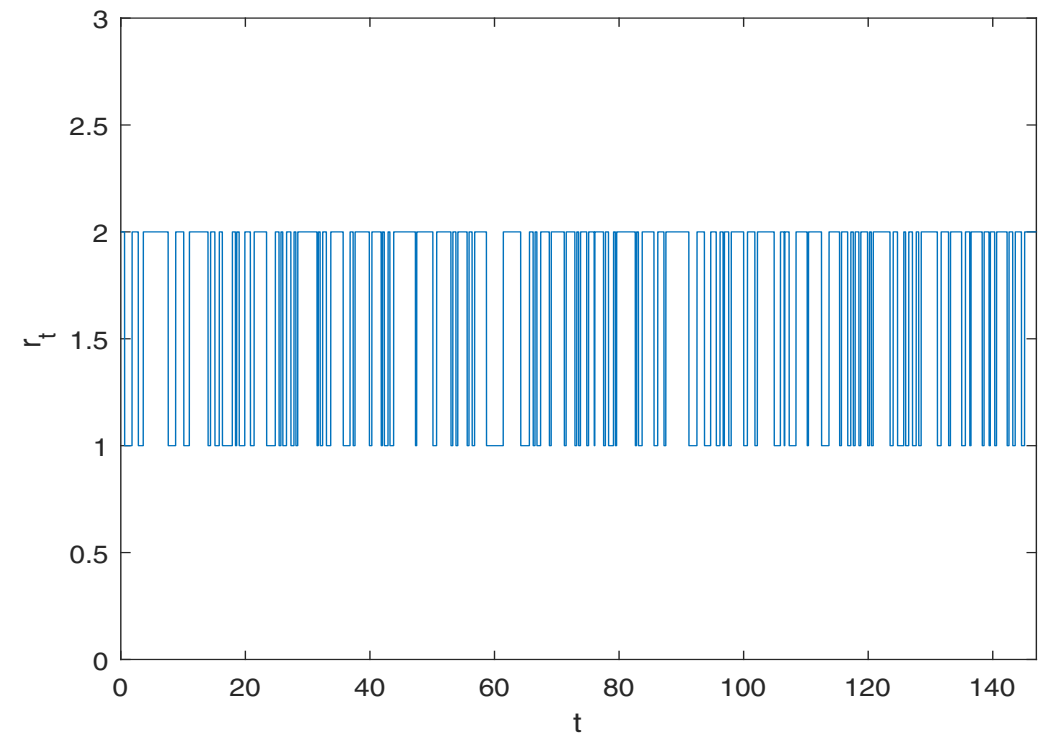

Figure 6: Response of $r_{t}$.

The desired controller are designed simultaneously. Finally, the effectiveness of the proposed sampled-data controller has been demonstrated by an illustrative example. It is noteworthy that the proposed method can be extended to the stochastic asynchronous and sliding model control $[44,45]$ of semi-Markovian jump chaotic Lur'e systems subject to multiple sampling periods. This will be the topic of our future study.

\section{Acknowledgment}

The authors would like to thank the editors and the reviewers for their valuable suggestions and comments which have led to a much improved paper. This work was supported by the National Natural Science Foundation of China under Grant(No. 61533006, 61703060, 11601474 and 11461082), Opening Fund of Geomathematics Key Laboratory of Sichuan Province (scsxdz201704), and Research fund for International Young Scientists of National Natural Science Foundation of China (NSFC Grant No. 61550110248), and NSERC Canada.

\section{Appendix}

$$
\begin{aligned}
\xi(t)= & {\left[e^{T}(t), \dot{e}^{T}(t), e^{T}\left(t_{l(j)}\right), e^{T}(t-\bar{\alpha} h), e^{T}(t-h), g^{T}(t), g^{T}(t-\bar{\alpha} h), g^{T}(t-h), \int_{t-\bar{\alpha} h}^{t} e^{T}(s) d s, \int_{t-h}^{t-\bar{\alpha} h} e^{T}(s) d s\right.} \\
& \left.\int_{t-\bar{\alpha} h}^{t} \int_{\theta}^{t} e^{T}(s) d s d \theta, \int_{t-h}^{t} \int_{\theta}^{t} e^{T}(s) d s d \theta, \zeta_{1}^{T}(t), \zeta_{2}^{T}(t), \ldots, \zeta_{m}^{T}(t)\right]^{T} \\
\zeta_{i}(t)= & {\left[e^{T}\left(t-\bar{\rho}_{i}(t)\right), e^{T}\left(t-\lambda_{i} \rho_{i}\right), \frac{1}{\bar{\rho}_{i}} \int_{t-\lambda_{i} \rho_{i}}^{t-\rho_{i-1}} e^{T}(s) d s\right]^{T} } \\
\tilde{e}_{i}^{T}= & {[0, \ldots, I, \ldots, 0] \quad(i=1,2, \ldots, 12+3 m) }
\end{aligned}
$$




$$
\begin{aligned}
& \check{\Upsilon}_{0}=2 \tilde{e}_{1} P_{q} \tilde{e}_{2}^{T}-\left[\tilde{e}_{1}, \tilde{e}_{3}\right] \mathcal{H}\left[\tilde{e}_{1}, \tilde{e}_{3}\right]^{T}+2\left[\tilde{e}_{1}, \tilde{e}_{2}, \tilde{e}_{3}\right] M^{T}\left[\tilde{e}_{1}-\tilde{e}_{3}\right]^{T}+\tilde{e}_{1} R_{2} \tilde{e}_{1}^{T}+\tilde{e}_{4}\left(R_{3}-R_{2}\right) \tilde{e}_{4}^{T} \\
& -\tilde{e}_{5} R_{3} \tilde{e}_{5}^{T}+\tilde{e}_{2}\left(\bar{\alpha} h R_{4}+(1-\bar{\alpha}) h R_{5}\right) \tilde{e}_{2}^{T}-\left[\tilde{e}_{1}, \tilde{e}_{4}, \tilde{e}_{9}\right]\left(\Omega_{1}\left(\mu_{1}, \mu_{2}, \bar{\alpha} h\right) \otimes R_{4}\right)\left[\tilde{e}_{1}, \tilde{e}_{4}, \tilde{e}_{9}\right]^{T} \\
& -\left[\tilde{e}_{4}, \tilde{e}_{5}, \tilde{e}_{10}\right]\left(\Omega_{1}\left(\mu_{3}, \mu_{4},(1-\bar{\alpha}) h\right) \otimes R_{5}\right)\left[\tilde{e}_{4}, \tilde{e}_{5}, \tilde{e}_{10}\right]^{T}+\tilde{e}_{2}\left(\frac{\bar{\alpha}^{4} h^{2}}{4} R_{6}+\frac{h^{2}}{4} R_{7}\right) \tilde{e}_{2}^{T} \\
& -\left[\tilde{e}_{1}, \tilde{e}_{9}, \tilde{e}_{11}\right]\left(\Omega_{2}(\bar{\alpha} h) \otimes R_{6}\right)\left[\tilde{e}_{1}, \tilde{e}_{9}, \tilde{e}_{11}\right]^{T}-\left[\tilde{e}_{1}, \tilde{e}_{9}+\tilde{e}_{10}, \tilde{e}_{12}\right]\left(\Omega_{2}(h) \otimes R_{7}\right)\left[\tilde{e}_{1}, \tilde{e}_{9}+\tilde{e}_{10}, \tilde{e}_{12}\right]^{T} \\
& +2 \tilde{e}_{2} D^{T}\left(L_{1}-L_{2}\right) \tilde{e}_{6}^{T}+2 \tilde{e}_{1} D^{T}\left(\bar{K}^{+} L_{2}-\bar{K}^{-} L_{1}\right) D \tilde{e}_{2}^{T}+\theta_{1} \tilde{e}_{1} Q_{1} \tilde{e}_{1}^{T}+\sum_{i=1}^{m-1} \tilde{e}_{11+3 i}\left(\theta_{i+1} Q_{i+1}-\theta_{i} Q_{i}\right) \tilde{e}_{11+3 i}^{T} \\
& -\theta_{m} \tilde{e}_{11+3 m} Q_{m} \tilde{e}_{11+3 m}^{T}+\sum_{i=1}^{m} \theta_{i} \bar{\rho}_{i}^{2} \tilde{e}_{2} Z_{i} \tilde{e}_{2}^{T}+\theta_{1}\left[\tilde{e}_{1}, \tilde{e}_{13}, \tilde{e}_{14}\right] \mathcal{S}_{11}\left[\tilde{e}_{1}, \tilde{e}_{13}, \tilde{e}_{14}\right]^{T} \\
& +\sum_{i=2}^{m} \theta_{i}\left[\tilde{e}_{8+3 i}, \tilde{e}_{10+3 i}, \tilde{e}_{11+3 i}\right] \mathcal{S}_{1 i}\left[\tilde{e}_{8+3 i}, \tilde{e}_{10+3 i}, \tilde{e}_{11+3 i}\right]^{T}+\theta_{1}\left[\tilde{e}_{1}, \tilde{e}_{14}, \tilde{e}_{15}\right] \mathcal{S}_{21}\left[\tilde{e}_{1}, \tilde{e}_{14}, \tilde{e}_{15}\right]^{T} \\
& +\sum_{i=2}^{m} \theta_{i}\left[\tilde{e}_{8+3 i}, \tilde{e}_{11+3 i}, \tilde{e}_{12+3 i}\right] \mathcal{S}_{2 i}\left[\tilde{e}_{8+3 i}, \tilde{e}_{11+3 i}, \tilde{e}_{12+3 i}\right]^{T} \\
& +2\left[y_{1} \tilde{e}_{2}+y_{2} \tilde{e}_{1}+y_{3} \tilde{e}_{3}\right]\left[-N \tilde{e}_{2}^{T}+N A_{q} \tilde{e}_{1}^{T}+N B_{q} \tilde{e}_{5}^{T}+N W_{q} \tilde{e}_{6}^{T}-\sum_{l=1}^{m} \theta_{l} U C_{q} \tilde{e}_{10+3 l}^{T}\right], \\
& \Upsilon_{0}=\check{\Upsilon}_{0}+\sum_{j=1}^{s} \pi_{q j}(h) \tilde{e}_{1} P_{j} \tilde{e}_{1}^{T}, \\
& \Upsilon_{01}=-2 \tilde{e}_{6} V_{11} \tilde{e}_{6}^{T}+2 \tilde{e}_{1} D^{T}\left[\bar{K}^{+}+\bar{K}^{-}\right] V_{11} \tilde{e}_{6}^{T}-2 \tilde{e}_{1} D^{T} \bar{K}^{+} V_{11} \bar{K}^{-} D \tilde{e}_{1}^{T}-2 \tilde{e}_{7} V_{21} \tilde{e}_{7}^{T}+2 \tilde{e}_{4} D^{T}\left[\bar{K}^{+}+\bar{K}^{-}\right] V_{21} \tilde{e}_{7}^{T} \\
& -2 \tilde{e}_{4} D^{T} \bar{K}^{+} V_{21} \bar{K}^{-} D \tilde{e}_{4}^{T}-2 \tilde{e}_{8} V_{31} \tilde{e}_{8}^{T}+2 \tilde{e}_{5} D^{T}\left[\bar{K}^{+}+\bar{K}^{-}\right] V_{31} \tilde{e}_{8}^{T}-2 \tilde{e}_{5} D^{T} \bar{K}^{+} V_{31} \bar{K}^{-} D \tilde{e}_{5}^{T}, \\
& \Upsilon_{02}=-2 \tilde{e}_{6} V_{12} \tilde{e}_{6}^{T}+2 \tilde{e}_{1} D^{T}\left[\bar{K}^{+}+\bar{K}^{-}\right] V_{12} \tilde{e}_{6}^{T}-2 \tilde{e}_{1} D^{T} \bar{K}^{+} V_{12} \bar{K}^{-} D \tilde{e}_{1}^{T}-2 \tilde{e}_{7} V_{22} \tilde{e}_{7}^{T}+2 \tilde{e}_{4} D^{T}\left[\bar{K}^{+}+\bar{K}^{-}\right] V_{22} \tilde{e}_{7}^{T} \\
& -2 \tilde{e}_{4} D^{T} \bar{K}^{+} V_{22} \bar{K}^{-} D \tilde{e}_{4}^{T}-2 \tilde{e}_{8} V_{32} \tilde{e}_{8}^{T}+2 \tilde{e}_{5} D^{T}\left[\bar{K}^{+}+\bar{K}^{-}\right] V_{32} \tilde{e}_{8}^{T}-2 \tilde{e}_{5} D^{T} \bar{K}^{+} V_{32} \bar{K}^{-} D \tilde{e}_{5}^{T}, \\
& \Upsilon_{1}(\hat{T})=2 \hat{T}\left[\tilde{e}_{1}, \tilde{e}_{3}\right] \mathcal{H}\left[\tilde{e}_{2}, 0\right]^{T}+\hat{T} \tilde{e}_{2} R_{1} \tilde{e}_{2}^{T} \quad\left(\hat{T}=0,(\bar{N}+1) \rho_{m}\right), \\
& \varepsilon^{T}(t)=\left[e^{T}(t), e^{T}\left(t_{l^{(j)}}\right)\right], \\
& \mathcal{H}=\left[\begin{array}{cc}
2 \delta\left(H_{1}\right)_{s} & -H_{1}+H_{2} \\
* & 2\left(-H_{2}+(1-\delta) H_{1}\right)_{s}
\end{array}\right], \\
& L_{1}=\operatorname{diag}\left\{\omega_{1}, \omega_{2}, \ldots, \omega_{m_{f}}\right\} \text {, } \\
& L_{2}=\operatorname{diag}\left\{\iota_{1}, \iota_{2}, \ldots, \iota_{m_{f}}\right\}, \\
& \bar{K}^{+}=\operatorname{diag}\left\{k_{1}^{+}, k_{2}^{+}, \ldots, k_{m_{f}}^{+}\right\}, \\
& \bar{K}^{-}=\operatorname{diag}\left\{k_{1}^{-}, k_{2}^{-}, \ldots, k_{m_{f}}^{-}\right\} \text {, } \\
& \varphi^{T}(t)=\left[e^{T}(t), \dot{e}^{T}(t), e^{T}\left(t_{l(j)}\right)\right], \\
& \Omega_{2}\left(x_{4}\right)=\left[\begin{array}{ccc}
\frac{3}{2} & 0 & \frac{-3}{x_{4}^{2}} \\
0 & \frac{3}{x_{4}^{2}} & \frac{-6}{x_{4}^{3}} \\
\frac{-3}{x_{4}^{2}} & \frac{-6}{x_{4}^{3}} & \frac{18}{x_{4}^{4}}
\end{array}\right] \text {, }
\end{aligned}
$$




$$
\begin{aligned}
& \eta_{1 i}^{T}=\left[e^{T}\left(t-\rho_{i-1}\right), e^{T}\left(t-\bar{\rho}_{i}(t)\right), e^{T}\left(t-\lambda_{i} \rho_{i}\right)\right], \\
& \eta_{2 i}^{T}=\left[e^{T}\left(t-\rho_{i-1}\right), e^{T}\left(t-\lambda_{i} \rho_{i}\right), \frac{1}{\bar{\rho}_{i}} \int_{t-\lambda_{i} \rho_{i}}^{t-\rho_{i-1}} e^{T}(s) d s\right], \\
& \mathcal{S}_{1 i}=\left[\begin{array}{ccc}
-S_{1 i} & S_{1 i}-\bar{W}_{i} & \bar{W}_{i} \\
* & -2 S_{1 i}+\bar{W}_{i}+\bar{W}_{i}^{T} & S_{1 i}-\bar{W}_{i} \\
* & * & -S_{1 i}
\end{array}\right], \\
& \mathcal{S}_{2 i}=\left[\begin{array}{ccc}
-\left(1+\frac{\pi^{2}}{4}\right) S_{2 i} & -\left(-1+\frac{\pi^{2}}{4}\right) S_{2 i} & \frac{\pi^{2}}{2} S_{2 i} \\
* & -\left(1+\frac{\pi^{2}}{4}\right) S_{2 i} & \frac{\pi^{2}}{2} S_{2 i} \\
* & * & -\pi^{2} S_{2 i}
\end{array}\right] \text {, } \\
& \left.\Gamma_{2}\left(\tilde{T}_{k+1+n_{j, j+1}}\right)\right\}=\Upsilon_{0}+\Upsilon_{02}+\tilde{T}_{k+1+n_{j, j+1}}\left[\tilde{e}_{1}, \tilde{e}_{2}, \tilde{e}_{3}\right] M^{T} R_{1}^{-1} M\left[\tilde{e}_{1}, \tilde{e}_{2}, \tilde{e}_{3}\right]^{T}, \\
& \mathcal{P}_{q}=\left[P_{1}-P_{q}, \ldots, P_{q-1}-P_{q}, P_{q+1}-P_{q}, \ldots, P_{s}-P_{q}\right], \\
& \mathcal{T}_{q}=\operatorname{diag}\left\{T_{q, 1}, \ldots, T_{q, q-1}, T_{q, q+1}, \ldots, T_{q, s}\right\}, \\
& \Upsilon_{0}^{*}=\check{\Upsilon}_{0}+\tilde{e}_{1}\left\{\sum_{j=1}^{s} \pi_{q j} P_{j}+\sum_{j=1, j \neq q}^{s}\left[\frac{\tilde{\pi}_{q j}^{2}}{4} T_{q j}\right\} \tilde{e}_{1}^{T},\right. \\
& \tilde{\Upsilon}_{0}^{*}=\check{\Upsilon}_{0}+\tilde{e}_{1}\left\{\sum_{j=1}^{s} \pi_{q j} P_{j}+\sum_{j=1, j \neq q}^{s}\left[\frac{\tilde{\pi}_{q j}^{2}}{4} T_{q j}+\left(P_{j}-P_{q}\right) T_{q j}^{-1}\left(P_{j}-P_{q}\right)\right]\right\} \tilde{e}_{1}^{T} .
\end{aligned}
$$

\section{References}

\section{References}

[1] Q. Zhu, J. Cao, pth moment exponential synchronization for stochastic delayed Cohen-Grossberg neural networks with Markovian switching, Nonlinear Dyn. 67 (1) (2012) 829-845.

[2] Q. Zhu, J. Cao, Adaptive synchronization under almost every initial data for stochastic neural networks with time-varying delays and distributed delays, Commun. Nonlinear Sci. Numer. Simul. 16 (4) (2011) 2139-2159.

[3] S. Zhang, X. Meng, T. Zhang, Dynamics analysis and numerical simulations of a stochastic non-autonomous predator-prey system with impulsive effects, Nonlinear Analysis: Hybrid Systems 26 (2017) 19-37.

[4] L. M. Pecora, T. L. Carroll, Synchronization in chaotic systems, Phys. Rev. Lett. 64 (8) (1990) 821-824.

[5] H. Zeng, Ju H. Park, S. Xiao, Y Liu, Further results on sampled-data control for master-slave synchronization of chaotic Lur'e systems with time delay, Nonlinear Dyn. 82 (2015) 851-863.

[6] Z. Wu, P. Shi, H. Su, J. Chu, Sampled-data synchronization of chaotic Lur'e systems with time delays, IEEE Trans. Neural Netw. Learn. Syst. 24 (3) (2013) 410-421.

[7] K. Shi, X. Liu, H. Zhu, S. Zhong, Y. Liu, C. Yin, Novel integral inequality approach on master-slave synchronization of chaotic delayed Lur'e systems with sampled-data feedback control, Nonlinear Dyn. 83 (3) (2016) 1259-1274.

[8] C. Hua, C. Ge, X. Guan, Synchronization of chaotic Lur'e systems with time delays using sampled-data control, IEEE T. Neur. Net. Lear. 26 (2015) 1214-1221.

[9] L. Zhang, Y. Leng, P. Colaneri, Stability and stabilization of discrete-time semi-Markov jump linear systems via semiMarkov kernel approach, IEEE Trans. Autom. Control 61 (2) (2016) 503-508.

[10] H. Shen, F. Li, S. Xu, V. Sreeram, Slow state variables feedback stabilization for semi-Markov jump systems with singular perturbations, IEEE Transactions on Automatic Control 63 (8) (2018) 2709-2714. 
[11] H. Shen, F. Li, H. Yan, H. R. Karimi, H.K. Lam, Finite-time event-triggered $H_{\infty}$ control for T-S fuzzy Markov jump systems, IEEE Transactions on Fuzzy Systems 26 (5) (2018) 3122-3135.

[12] J. Cheng, J.H. Park, J. Cao, W. Qi, Hidden Markov model-based nonfragile state estimation of switched neural network with probabilistic quantized outputs, IEEE Transactions on Cybernetics, DOI:10.1109/TSMC.2018.2866906.

[13] J. Cheng, J.H. Park, H.R. Karimi, H. Shen, A flexible terminal approach to sampled-data exponentially synchronization of Markovian neural networks with time-varying delayed signals, IEEE Trans Cybern 48 (8) (2018) 2232-2244.

[14] M. Luo, G. Liu, S. Zhong, Robust fault detection of Markovian jump systems with different system modes, Appl. Math. Model. 37 (2013) 5001-5012.

[15] Q. Zhu, Stability analysis of stochastic delay differential equations with lévy noise, Syst. Control Lett. 118 (2018) 62-68.

[16] B. Wang, Q. Zhu, Stability analysis of Markov switched stochastic differential equations with both stable and unstable subsystems, Syst. Control Lett. 105 (2017), 55-61.

[17] Q. Zhu, Q. Zhang, pth moment exponential stabilisation of hybrid stochastic differential equations by feedback controls based on discrete-time state observations with a time delay, Iet. Control Theory. A. 11 (12) (2017) 1992-2003.

[18] B. Wang, Q. Zhu, Stability analysis of semi-Markov switched stochastic systems, Automatica 94 (2018) 72-80.

[19] J. Cheng, C. K. Ahn, H. R. Karimi, J. Cao, W. Qi, An event-based asynchronous approach to Markov jump systems with hidden mode detections and missing measurements, IEEE Trans. Syst., Man, Cybern., Syst.(2018) DOI: 10.1109/TSMC.2018.2866906.

[20] B. Wang, D. Zhang, J. Cheng, J.H. Park, Fuzzy model-based nonfragile control of switched discrete-time systems, Nonlinear Dynamics 93 (4) (2018) 2461-2471.

[21] J Xia, G Chen, W Sun, Extended dissipative analysis of generalized Markovian switching neural networks with two delay components, Neurocomputing 260 (2017) 275-283.

[22] G Chen, J Xia, G Zhuang, Delay-dependent stability and dissipativity analysis of generalized neural networks with Markovian jump parameters and two delay components, J. Frankl. Inst. 353 (9) (2016) 2137-2158.

[23] G Chen, J Xia, G Zhuang, Improved passivity analysis for neural networks with Markovian jumping parameters and interval time-varying delays, Neurocomputing 155 (2015) 253-260.

[24] X. Meng, L. Zhang, Evolutionary dynamics in a Lotka-Volterra competition model with impulsive periodic disturbance, Mathematical Methods in the Applied Sciences 39 (2) (2016) 177-188.

[25] L. Su, H. Shen, Mixed $H_{\infty}$ passive synchronization for complex dynamical networks with sampled-data control, Appl. Math. Comput. 259 (2015) 931-942 .

[26] F. Qiu, J. Cao, Exponential stability and $l_{2}$-gain analysis for sampled-data control of linear systems, J. Frankl. Inst. 353 (2016) 462-477.

[27] J. Cheng, S. Chen, Z. Liu, H. Wang, J. Li, Robust finite-time sampled-data control of linear systems subject to random occurring delays and its application to Four-Tank system, Appl. Math. Comput. 281 (2016) 55-76.

[28] W. Kang, J. Cheng, B. Wang, Ju H. Park, H.M. Fardoun, Event-triggered reliable control for Markovian jump systems subject to nonuniform sampled data, J. Franklin. I. 354 (2017) 5877-5894.

[29] B. Bamieh, J. Pearson, B. Francis, A. Tannenbaum, A lifting technique for linear periodic systems with applications to sampled-data control. Syst. Control Lett. 17 (1991) 79-88.

[30] E. Fridman, A refined input delay approach to sampled-data control, Automatica 46 (2010) 421-427.

[31] S. Xu, T. Chen, Robust $H_{\infty}$ filtering for uncertain impulsive stochastic systems under sampled measurements, Automatica 39 (2003) 509-516.

[32] C. Wen, A. Qiu, B. Jiang, An output delay approach to fault estimation for sampled-data systems. Sci. China Inf. Sci. 55 (2012) 2128-2138.

[33] J. P. Hespanha, P. Naghshtabrizi, Y. Xu, A survey of recent results in networked control systems, Proc. IEEE, 95 (1) 
(2007) 138-162.

[34] Y. Zhang, H. Fang, Stabilization of nonlinear networked systems with sensor random packet dropout and time-varying delay, Appl. Math. Model. 35 (2011) 2253-2264.

[35] Z. Hu, F. Deng, Modeling and stabilization of networked control systems with bounded packet dropouts and occasionally missing control inputs subject to multiple sampling periods, J. Frankl. Inst. 354 (2017) 4675-4696.

[36] H. Gao, J. Wu, P. Shi, Robust sampled-data $H_{\infty}$ control with stochastic sampling, Automatica 45 (7) (2009) $1729-1736$.

[37] P. Park, J.W. Ko, C. Jeong, Reciprocally convex approach to stability of systems with time-varying delays, Automatica 47 (1) (2011) 235-238.

[38] F. Li, L. Wu, P. Shi, Stochastic stability of semi-Markovian jump systems with mode-dependent delays, Int. J. Robust. Nonlin. 24 (2014) 3317-3330.

[39] Q. Zhu, Stabilization of stochastic nonlinear delay systems with exogenous disturbances and the event-triggered feedback control, IEEE T. Automat. Contr (2018, in press) DOI: 10.1109/TAC.2018.2882067.

[40] Y. Wei, J. Qiu, H. Lam, A novel approach to reliable output feedback control of fuzzy-affine systems with time delays and sensor faults, IEEE T. Fuzzy Syst. 25 (6) (2017) 1808-1823.

[41] Y. Wei, J. H. Park, J. Qiu, L. Wu, H. Y. Jung, Sliding mode control for semi-Markovian jump systems via output feedback, Automatica 81 (2017) 133-141.

[42] Y. Wei, J. Qiu, P. Shi, L. Wu, A piecewise-Markovian Lyapunov approach to reliable output feedback control for fuzzyaffine systems with time-delays and actuator faults, IEEE Trans. Cybern. 48 (2018) 2723-2735.

[43] Y. Wei, J. Qiu, H. R. Karimi, W. Ji, A novel memory filtering design for semi-Markovian jump time-delay systems, IEEE Trans. Syst., Man, Cybern., Syst. 48 (2018) 2229-2241.

[44] S. Ding, W.X. Zheng, J. Sun, J. Wang, Second-order sliding mode controller design and its implementation for buck converters, IEEE Transactions on Industrial Information 14 (5) (2018) 1990-2000.

[45] S. Ding, S. Li, Second-order sliding mode controller design subject to mismatched term, Automatica 77 (2017) $388-392$. 